\title{
Flora del bosque tropical caducifolio en una zona con suelos yesosos y calcáreos de Colima, México
}

\section{Flora of the tropical deciduous forest in a gypseous and calcareous area, Colima, Mexico}

\section{Acta Botanica Mexicana}

\author{
Mollie Harker' (D, Leticia Hernández-López² (D), Miguel Ángel Muñiz-Castro' (1)
}

\section{Resumen}

Antecedentes y Objetivos: La microcuenca La Salada, ubicada al centro-sur de Colima, cubierta por bosque tropical caducifolio (BTC) sobre sustratos yesosos (74.5\%) y calcáreos (25.5\%), fue explorada por el eminente botánico Rogers McVaugh para la Flora Novo-Galiciana. Sin embargo, solo parte del material colectado ha estado disponible. El objetivo del trabajo fue documentar la diversidad de plantas vasculares de la zona, para evidenciar su relevancia biológica.

Métodos: Se realizaron una revisión bibliográfica y de material de herbario (IBUG y MICH) y colectas de campo para integrar la lista florística. Complementariamente se revisaron bases de datos electrónicas (IBdata y GBIF) para obtener registros de colectas en sitios de Colima con características ecológicas similares al área muestreada. Para comparar la diversidad del área con la de otros sitios con BTC se calculó el índice de diversidad taxonómica (IDT).

Resultados clave: Se registraron 408 taxones, 248 géneros y 78 familias de la flora de BTC de sustratos yesoso-calcáreos de Colima. Se presentan dos apéndices, uno se basa en registros del área muestreada por los autores y otro en registros de otras áreas yesoso-calcáreas de Colima obtenidos de bases de datos. Del área muestreada se registraron 368 taxones (incluyendo 16 infraespecíficos) de 233 géneros y 75 familias. Fabaceae, Euphorbiaceae, Asteraceae, Malvaceae, Poaceae y Convolvulaceae son las familias más representadas (48\% de los taxones). Los géneros más diversos son Euphorbia (13), Bursera (ocho), Ipomoea (siete) y Tillandsia (seis). De las especies registradas 39\% (144) son endémicas de México y el área constituye la localidad tipo de 23. Se anotan 11 taxones no registrados antes para el estado de Colima. El valor IDT fue de 330.35 especies/In área.

Conclusiones: La alta diversidad florística y endemismo de la zona, y la singularidad del sustrato yesoso, merecen la conservación de estos ecosistemas que están siendo impactados en Colima.

Palabras clave: biodiversidad, conservación, diversidad taxonómica, McVaugh, selvas estacionalmente secas, Sierra Madre del Sur.

\section{Abstract}

Background and Aims: The study area of La Salada basin is situated in the southern-central area of Colima, characterized by tropical dry forest vegetation on gypseous (74.5\%) and calcareous (25.5\%) soils. This site was visited by the eminent botanist Rogers McVaugh and is represented in the Flora Novo-Galiciana. However, only a part of his collections has been available for consultation. The objective here is to document the diversity of vascular plants in this site providing evidence which sustains its biological relevance.

Methods: Revisions of bibliography and material in the herbaria IBUG and MICH were made. Specimens from recent fieldwork were integrated into the list. Additionally, electronic databases (IBdata and GBIF) were reviewed to obtain records of collections in sites of Colima with ecological characteristics similar to the sampled area. The Index of Taxonomic Diversity (IDT) was calculated facilitating comparisons of values for other areas with tropical dry forests.

Key results: A total of 408 taxa, 248 genera and 78 families of vascular plants of BTC in gypseous-calcareous soils of Colima were registered. Two appendices are provided: the first with authors' collections and those in bibliography; and the second with citations of species in other gypseous-calcareous soils in Colima from databases. In the first, 368 taxa (including 16 infraspecific taxa) in 233 genera and 75 families were recorded. The families Fabaceae, Euphorbiaceae, Asteraceae, Malvaceae, Poaceae and Convolvulaceae total $48 \%$ of the reported flora. The genera with greatest diversity were Euphorbia (13), Bursera (eight), Ipomoea (seven) and Tillandsia (six). Of the species recorded 39\% (144) are endemic to Mexico and this site is the type location for 23 species. The IDT calculated is 330.35 species/In area.

Conclusions: The high floristic diversity and endemism of the area with gypseous and calcareous soils, substantiate conservation of these ecosystems which are being impacted in Colima.

Key words: biodiversity, conservation, McVaugh, taxonomic diversity, tropical seasonally dry forests, Sierra Madre del Sur.

${ }^{1}$ Universidad de Guadalajara, Centro Universitario Recibido: 8 de noviembre de 2020. de Ciencias Biológicas y Agropecuarias (CUCBA), Revisado: 8 de diciembre de 2020.

Departamento de Botánica y Zoología, Camino Ing. Aceptado por Marie-Stéphanie Samain: 20 de marzo Ramón Padilla Sánchez 2100, 45200 Las Agujas, Zapo- de 2021. pan, Jalisco, México.

${ }^{2}$ Autor para la correspondencia: leticia.hernandez@ academicos.udg.mx
Citar como: Harker, M., L. Hernández-López y M. Á Muñiz-Castro. 2021. Flora del bosque tropical caducifolio en una zona con suelos yesosos y calcáreos de Colima, México. Acta Botanica Mexicana 128: e1818. DOI: https://doi.org/10.21829/abm128.2021.1818 


\section{Introducción}

Los bosques tropicales estacionalmente secos, denominados en México como bosques tropicales caducifolios (BTC) sensu Rzedowski (1978), o selvas bajas caducifolias sensu Miranda y Hernández-X. (1963), se encuentran en el mundo principalmente entre $20^{\circ}$ y $10^{\circ}$ de latitud norte $y$ sur; en América cubren desde el noroeste de México y Las Antillas, hasta el norte de Argentina (Bezaury, 2010; CebaIlos y Valenzuela, 2010). En México, el BTC se distribuye desde Sonora y el sur de la Península de Baja California, hasta Chiapas; cubre $\mathbf{7 . 6 \%}$ de la superficie nacional (Trejo, 2010).

En términos generales, el BTC se define como una comunidad donde predominan los árboles de baja altura con dosel cerrado y la mayor parte de las plantas pierden las hojas en la época seca, la que puede durar hasta siete u ocho meses (Rzedowski y McVaugh, 1966; Gentry, 1995; Trejo, 2010; DRYFLOR, 2016). Se desarrolla en zonas desde el nivel del mar hasta $1700 \mathrm{~m}$ de altitud, con temperatura media anual mayor a $20^{\circ} \mathrm{C}$ y una precipitación pluvial anual entre 700 y 1200 mm (Arévalo et al., 2016). Crece sobre laderas con suelos someros, pedregosos, sobre afloramientos tanto ígneos como metamórficos $y$, en no pocas ocasiones, sobre sustratos sedimentarios marinos (Rzedowski, 1978) como los calizos y yesosos.

A nivel de comunidades vegetales, el BTC ha sido objeto de gran cantidad de estudios desde diferentes perspectivas (Rzedowski y McVaugh, 1966; Rzedowski, 1978; Lott, 1985, 1993; Cuevas-Guzmán et al., 1998; Ceballos et al., 2010). Todos indican que este ecosistema alberga alto endemismo y riqueza de especies. Al mismo tiempo, resaltan la alta transformación en el uso del suelo, por lo que su conservación debería considerarse como prioritaria empleando diferentes estrategias (Ceballos y García 1995; Bezaury, 2010; Lott y Atkinson, 2010; Sousa, 2010; BáezMontes, 2016b).

Los BTC de la vertiente del Pacífico de México presentan una de las mayores diversidades de flora del continente americano, además de un considerable endemismo (Gentry, 1995; Lott y Atkinson, 2010). En especial las ecorregiones de BTC de Jalisco y el Balsas son consideradas como las de más alta prioridad para la conservación a una escala regional (Dinerstein et al., 1995; Bezaury, 2010). Gentry
(1995) considera los BTC del occidente de México entre los más secos de los bosques neotropicales. En los estados de Colima, Jalisco y Nayarit, el BTC ocupa grandes extensiones de terreno entre 0 y $1600 \mathrm{~m}$ de altitud (Rzedowski, 1978). Dentro de estos BTC prioritarios se encuentran zonas únicas en sus características geológicas y edafológicas que pueden aportar un mayor valor al grado de endemismo, como son las zonas con afloramientos yesosos y calcáreos de origen Cretácico de la Sierra Madre del Sur en los estados de Jalisco, Colima, Michoacán, Guerrero y Oaxaca (SGM, 2019). Así mismo, García (2010) enfatiza la necesidad de conservar las selvas secas (BTC) del centro del estado de Colima por su alta diversidad y endemismo de flora y fauna, zona que coincide en parte con afloramientos calcáreos y yesosos (SGM, 2010).

Colima significa "lugar donde domina el Dios del fuego" y probablemente alude a la presencia del Volcán de Fuego de Colima (Báez-Montes, 2016a). El estado de Colima está situado en el occidente de México, donde colindan las provincias biogeográficas Faja Volcánica Transmexicana, Sierra Madre del Sur y Tierras Bajas del Pacífico (Morrone et al., 2017). El clima predominante en la zona costera y en el valle de Tecomán (al sur del estado) es cálido subhúmedo y cambia a cálido semiseco y seco en la zona central del estado, en la transición de las llanuras hacia las sierras, hasta alcanzar el templado en el volcán de Colima, al norte del estado (Castro-Caro, 2016). La variación altitudinal, que va de 0 a $4225 \mathrm{~m}$, entre otras condiciones, propicia el desarrollo de 14 tipos de vegetación (Arévalo et al., 2016). Destaca el bosque tropical caducifolio (BTC), por ser el tipo de vegetación nativa más extenso en la entidad (Rzedowski, 1978; INEGI, 2017). En cuanto al conocimiento de la flora para el estado de Colima, Villaseñor (2016) registra 219 familias, 1267 géneros y 4333 especies. La obra Flora Novo-Galiciana incluye numerosas colecciones del estado, pero principalmente del área de la microcuenca de La Salada (McVaugh, 1983, 1984, 1985, 1987, 1989, 1992, 1993, 2001). Por su parte, Santana-Michel et al. (2016) documentaron las plantas de importancia apícola en este estado, listando 311 taxones. Padilla-Velarde et al. (2006) inventariaron la flora arbórea estatal dando cuenta de 550 especies. Otro trabajo que hace énfasis en especies arbóreas y que incide en particular en la zona calcárea-yesosa con 
BTC del centro-sur del estado, es el de Moreno-Gómez et al. (2016), quienes registran 65 especies de la microcuenca La Salada.

La microcuenca La Salada, ubicada a $17 \mathrm{~km}$ al sur de la ciudad de Colima, es una región de lomeríos y serranías bajas de clima tropical estacional, cuya particularidad florística más importante es la de presentar varias especies con un endemismo restringido. El tipo de vegetación que cubre esta zona es el BTC (Moreno-Gómez et al., 2016). Varios estudios incluyen la descripción de especies nuevas, cuyo espécimen tipo fue colectado en esta misma microcuenca, tales como Pinguicula colimensis McVaugh \& Mickel (McVaugh y Mickel, 1963), Lamourouxia colimae W.R. Ernst \& Baad (Ernst y Baad, 1970), Tetramerium mcvaughii T.F. Daniel (Daniel, 1986), Aristolochia colimensis Santana Mich. (Santana-Michel, 2002), Colima convoluta (Ravenna) Aarón Rodr. \& Ortiz-Catedral (Rodríguez y Ortiz-Catedral, 2003) y Agave pablocarrilloi A. Vázquez, Muñiz-Castro \& PadillaLepe (Vázquez-García et al., 2013). Incluso se incluye para la zona un género nuevo, Colima (Ravenna) Aarón Rodr. \& Ortiz-Catedral (Rodríguez y Ortiz-Catedral, 2003).

Rogers McVaugh recolectó, en seis ocasiones, en la zona de la microcuenca La Salada, entre 1957 y 1970 para la elaboración de la Flora Novo-Galiciana (McVaugh, 1972). Sin embargo, no se conoce parte de ese material botánico, debido a que no se depositaron duplicados en herbarios nacionales y/o no se ha publicado una lista exhaustiva de sus exsiccata. Sin embargo, parte de ese material colectado sí está citado en los volúmenes de la Flora Novo-Galiciana. Después de las exploraciones de McVaugh, los estudios botánicos en el área de estudio han sido escasos.

Otra de las particularidades ambientales de la microcuenca La Salada es que su vegetación de BTC se desarrolla en la zona de afloramientos yesosos más extensa de la entidad, aislados entre una matriz de suelos calcáreos (SGM, 2010). Es conocido que los suelos yesosos ejercen una presión de selección alta sobre las especies vegetales, debido a la menor disponibilidad de nutrientes, alta concentración de sulfatos y diferencias en la capacidad de retención de agua (Meyer et al., 1992; Ruiz et al., 2003; Palacio et al., 2007), lo que a su vez puede generar cambios fenológicos que pueden conducir a aislamientos reproductivos entre poblaciones y mayores tasas de especiación y endemis- mo (Meyer, 1986; Palacio et al., 2007). Hasta el momento, para la región mexicana con presencia de BTC se ha reportado una sola zona con afloramientos yesoso-calcáreos correspondiente a la meseta de Zoh-Laguna, en Calakmul, Campeche (Martínez et al., 2001; Martínez y Galindo-Leal, 2002). Sin embargo, en estos estudios no se analizó en forma separada la diversidad florística correspondiente al BTC de suelo yesoso, sino que se reportó la flora en forma general del total de diez tipos de vegetación, siendo el dominante la selva mediana subperennifolia.

Los inventarios biológicos sintetizan gran cantidad de información y constituyen la base para estudios ecológicos, biogeográficos, etnobotánicos, filogenéticos y de conservación de la biodiversidad. Por lo tanto, los objetivos del presente trabajo fueron: determinar la composición y diversidad de plantas vasculares del bosque tropical estacionalmente seco en una zona cálida con suelos yesosos relictuales del occidente de México, que además tiene valor histórico-botánico, y definir su importancia en endemismos.

\section{Materiales y Métodos}

\section{Área de estudio}

El área de estudio se ubica en el centro-sur del estado de Colima, México, e incluye una parte del suroeste del municipio Colima y la parte norte del municipio Tecomán. Se delimitó como el área cubierta por BTC con sustrato principalmente yesoso donde R. McVaugh realizó sus colectas históricas (McVaugh, 1983, 1984, 1985, 1987, 1989, 1992, 1993, 2001) (Fig. 1).

Esta área, correspondiente en su gran mayoría a la cabecera de la microcuenca La Salada, se encuentra aproximadamente entre los km 17 a 22 al sur de la ciudad de Colima, en las cercanías de las carreteras de cuota (110D) y la libre Colima - Manzanillo (110), entre los paralelos $19^{\circ} 3^{\prime}$ y $19^{\circ} 5.3^{\prime} \mathrm{N}$ y los meridianos $103^{\circ} 46^{\prime}$ y $103^{\circ} 48^{\prime} \mathrm{O}$. El área ocupa una superficie de 292 hectáreas correspondiente al polígono mínimo convexo que incluyó a todos los puntos de colecta con un búfer de $30 \mathrm{~m}$ alrededor de cada punto (Fig. 1); con un rango de elevación entre 370 y $600 \mathrm{~m}$. El clima es cálido subhúmedo con una temperatura media de $22^{\circ} \mathrm{C}$ en el mes más cálido y de $18{ }^{\circ} \mathrm{C}$ en el mes más frío. La precipitación acumulada media anual oscila entre 800 y $1200 \mathrm{~mm}$ (CONABIO, 2019). 


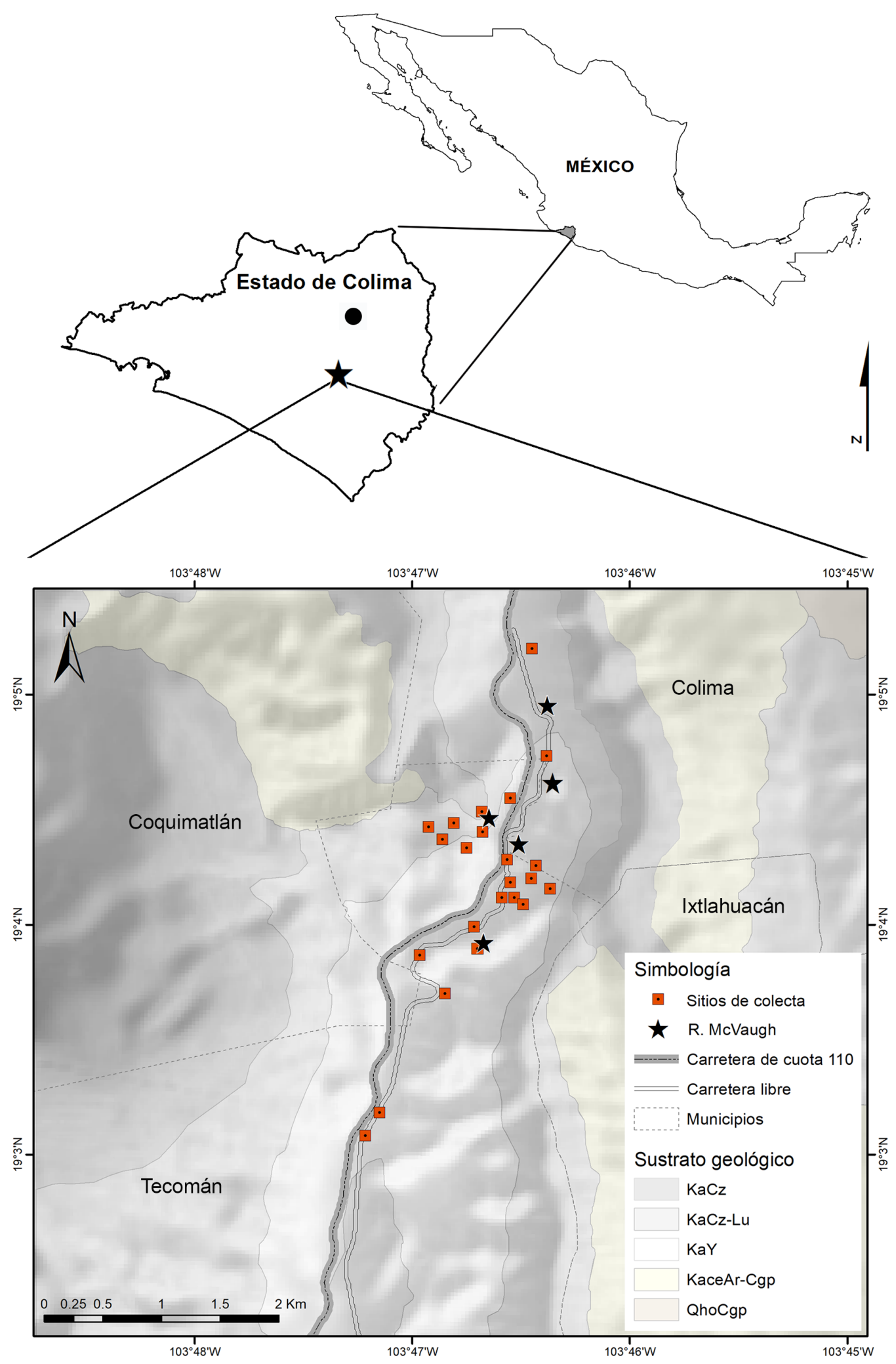

Figura 1: Ubicación del área de estudio en Colima, México (en el recuadro, los cuadros rojos indican sitios de colecta realizada por los autores y las estrellas señalan sitios de colecta histórica de R. McVaugh). El círculo sólido representa la ciudad de Colima. Los símbolos de sustratos geológicos son: $\mathrm{KaCz}=$ Caliza del Cretácico Inferior (piso Albiano), KaCz-Lu=Caliza con Lutita del Cretácico Inferior (Albiano), KaY= Yeso del Cretácico Inferior (Albiano), KaceAr-Cgp=Arenisca Conglomerado polimíctico del Cretácico Inferior (Albiano-Cenomiano) y Qho-Cgp=Conglomerado polimíctico del Cuaternario (Holoceno). Fuentes: CONABIO (2004), SGM (2010), INEGI (2013). 
La zona forma parte de la provincia biogeográfica Tierras Bajas del Pacífico (Morrone et al., 2017; Morrone, 2019) y de la ecorregión Bosque Tropical Caducifolio de Jalisco (Olson et al., 2001). Desde la perspectiva fisiográfica, se ubica en la subprovincia Cordillera Costera del Sur (Cervantes-Zamora et al., 1990), en la provincia Sierra Madre del Sur (INEGI, 2001). La mayor parte del área está conformada por lomeríos y sierras bajas de la microcuenca del Arroyo La Salada, y una pequeña parte del norte del área pertenece a la microcuenca del Arroyo Colima. Predominan los suelos del tipo litosol y en menor proporción regosol calcárico y vertisol pélico (CONABIO, 2019). La vegetación corresponde al bosque tropical caducifolio
(Rzedowski, 1978), (Fig. 2). El sustrato geológico del área de estudio está constituido por $\mathbf{7 4 . 5 \%}$ de afloramiento de yeso, correspondiente a las partes bajas de la cabecera de la microcuenca La Salada y $25.5 \%$ de caliza y calizalutita, el cual está representado por las laderas bajas de la cuenca, de los cerros Alcomún, La Media Luna, Galindo y La Cuesta de La Salada (SGM, 2010). Los afloramientos yesosos en el occidente de México son muy escasos, aislados y restringidos a pequeños parches entre matrices de rocas calcáreas. En el estado de Colima, la zona yesosa de la microcuenca La Salada (municipios Tecomán y Colima) representa la mayor parte de este tipo de sustrato para el estado. Aparte de esta zona, solo hay cuatro pequeños
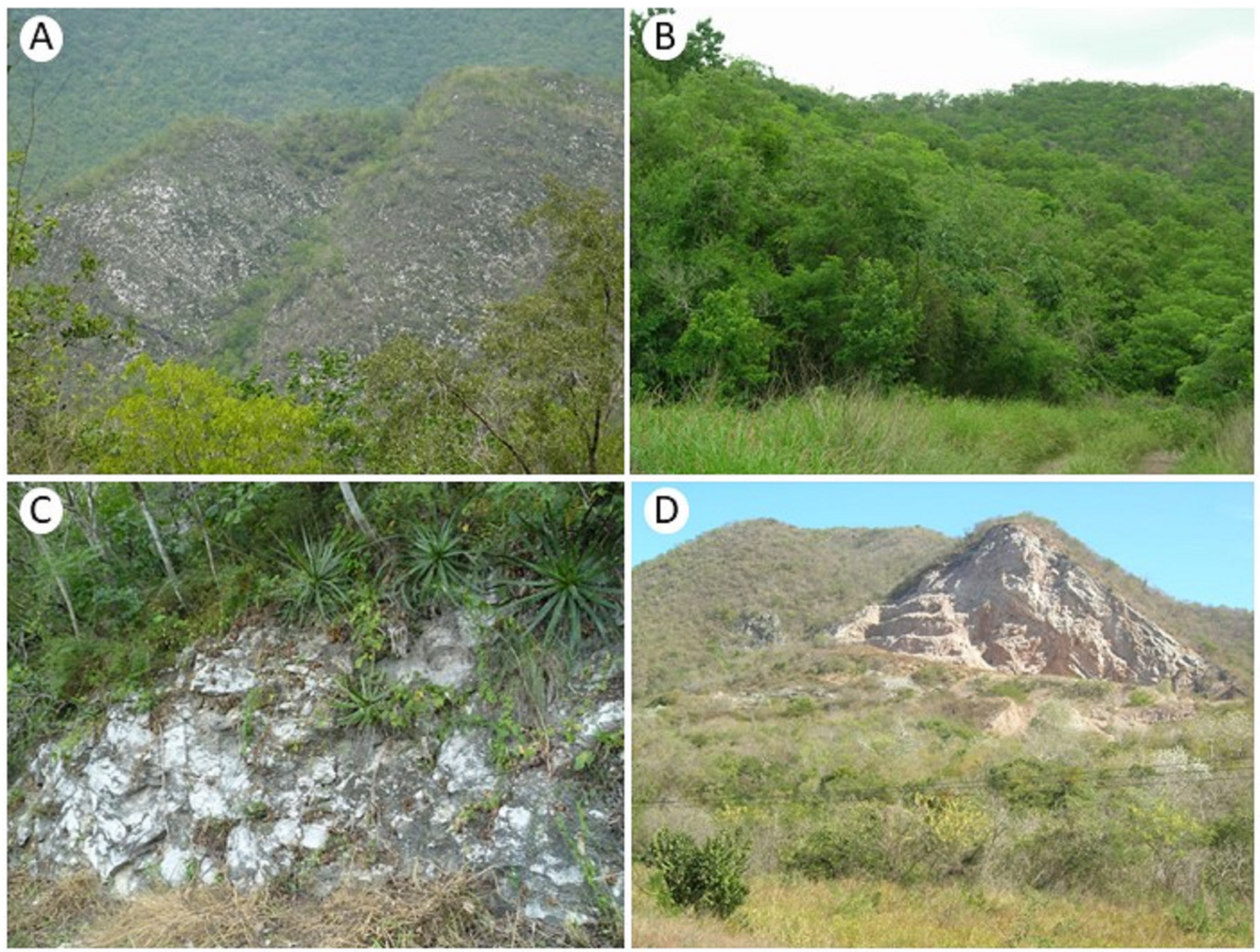

Figura 2: A. aspecto general del área de estudio; B. bosque tropical caducifolio en época de lluvias; C. ladera que muestra el sustrato calcáreo; D. banco de material para la elaboración de cal. Fotografías de M. Harker. 
afloramientos yesosos (en Agua Zarca, Cerro La Guelica, Cerro la Vieja y Cerro Atravesado), en el municipio vecino de Coquimatlán, que en su conjunto no superan la extensión de suelos yesosos de La Salada.

El área es de interés por una combinación de factores: sustrato yesoso y calcáreo, bosque tropical caducifolio en buen estado de conservación, con alta riqueza de especies y de endemismos, y relevancia en el contexto botánico (localidad tipo de varias especies).

\section{Elaboración del listado florístico}

Se revisaron los volúmenes de la Flora Novo-Galiciana (McVaugh, 1983, 1984, 1985, 1987, 1989, 1992, 1993, 2001) para obtener registros de las especies citadas para la zona. También se consultaron otras obras que incluyen inventarios florísticos de Colima (McVaugh, 1972; PadillaVelarde et al., 2006; CONABIO, 2016; Moreno-Gómez et al., 2016; Villaseñor, 2016). Con el fin de incluir otras colectas de Rogers McVaugh, no citadas en publicaciones previas, se revisaron sus libros de colecta y el herbario de la Universidad de Michigan (MICH) en los Estados Unidos de América. Así mismo, se revisaron bases de datos electrónicas para corroborar la nomenclatura actual de los ejemplares históricos de McVaugh (IBUNAM, 2018).

Para obtener información de colectas botánicas realizadas en sitios yesosos y calcáreos en los municipios Coquimatlán, Colima y Tecomán, se consultó la plataforma IBdata del Herbario Nacional del Instituto de Biología (MEXU) de la Universidad Nacional Autónoma de México (IBUNAM, 2021) y la base de datos de Global Biodiversity Information Facility (GBIF, 2021). Los registros obtenidos se depuraron y actualizaron en cuanto a la nomenclatura.

Se realizó trabajo de campo en varios periodos con nueve salidas en diferentes estaciones del año (febrero de 2002 , agosto y octubre de 2006 , enero y abril de 2007 y octubre de 2017). Se siguió el procedimiento tradicional para la preparación de material botánico (Lot y Chiang, 1986) y los ejemplares se depositaron en el Herbario Luz María ViIlareal de Puga (IBUG) de la Universidad de Guadalajara y se enviaron duplicados a los herbarios IEB y MEXU. Los acrónimos de los herbarios se listan con base en Thiers (2020). La información recabada se incorporó en una base de datos para el análisis posterior.
La distribución de las especies se determinó con base en la consulta de TROPICOS (TROPICOS, 2019) y de revisión bibliográfica (Villaseñor, 2016). La distribución de especies con respecto a los suelos yesosos y calcáreos se obtuvo a partir de la revisión de literatura (Rzedowski, 1978; Meyer et al., 1992; Hinton y Hinton, 1995).

El listado de especies se ordenó con base en APG IV (2016) para las angiospermas y según Pteridophyte Phylogeny Group (PPG I, 2016), para los helechos. En el listado se anotan la forma biológica (árbol, arbusto, hierba, trepadora, parásita), nombre científico, autores y nombres de colectores (abreviados) con su respectivo número de colecta. Los nombres científicos se revisaron en la plataforma Taxonomic Name Resolution Service (Boyle et al., 2013; TNRS, 2020) y TROPICOS (TROPICOS, 2019).

\section{Determinación de tipos de sustratos del área}

Para determinar de forma más precisa los sustratos geológicos del área de estudio y la elaboración de la figura 1, se utilizaron las siguientes fuentes: la carta geológica de Colima escala 1: 50,000 (SGM, 2010), el mapa base del estado de Colima (CONABIO, 2004) y el Continuo de Elevaciones Mexicano (CEM 3.0) de INEGI (2013).

\section{Estimación de diversidad taxonómica}

Con el fin de comparar la diversidad del área de estudio con la de otras áreas con BTC representativas del territorio mexicano que tienen suelos calcáreos y otros tipos de suelos y sustratos geológicos, se calculó el índice de diversidad taxonómica (IDT) propuesto por Squeo et al. (1998). En el cálculo del IDT se asume que el número de taxones depende del área de muestreo en forma logarítmica, y su fórmula es: IDT= $n_{i} / \ln A_{i}$, donde $n_{i}$ es el número de taxa, $y$ In $A_{i}$ es el logaritmo natural del área de muestreo de la unidad vegetal $\left(\mathrm{en} \mathrm{km}^{2}\right)$. La superficie del área de estudio se estimó como el polígono mínimo convexo que incluyó todos los puntos de colecta, tomando en cuenta una distancia de $30 \mathrm{~m}$ alrededor de los registros de colecta marcados con GPS (Garmin eTrex 30), distancia que equivale al alcance visual en las colectas. El cálculo del IDT no incluye el área de pequeños afloramientos yesosos correspondiente al municipio Coquimatlán, que no ha sido muestreada, por sus escasas dimensiones. 


\section{Resultados}

Se registraron 408 taxones, 248 géneros y 78 familias de la flora de BTC de sustratos yesoso-calcáreos de Colima. Los resultados se presentan en dos apéndices. En el Apéndice 1 se enlistan los registros del área de estudio muestreada por los autores (la microcuenca La Salada), este apéndice representa $90.2 \%$ de los taxones registrados y tanto el análisis florístico como el de diversidad se centran en estos datos; en el Apéndice 2 se presentan los registros de especies obtenidos de bases de datos electrónicas sobre colectas realizadas en la reducida superficie de BTC en suelos yesosocalcáreos del municipio Coquimatlán, Colima. Este incluye $9.8 \%$ del total de las especies.

Del área de estudio de la microcuenca La Salada (Apéndice 1) se registraron 368 taxones (incluidos 16 de categorías intraespecíficas) agrupados en 233 géneros y 75 familias (Cuadro 1). Las familias más diversas fueron Fabaceae, Euphorbiaceae, Asteraceae, Malvaceae, Poaceae y Convolvulaceae (Cuadro 2), las cuales conforman $48 \%$ del total de las especies aquí reportadas. Algunas especies representativas se muestran en la figura 3. Los géneros con mayor número de especies fueron Euphorbia L. (13), Bursera Jacq. ex L. (ocho), Ipomoea L. (siete) y Tillandsia L. (seis). Se listan nueve taxones hasta nivel de género, se trata de dos en proceso de descripción por los especialistas y otros que no se lograron determinar a nivel específico.

Se presentan novedades en este estudio que incluyen 11 taxones (uno de categoría infraespecífica) de 10 familias, que en la bibliografía consultada no se habían registrado para el estado de Colima. De ellas tres son árboles, un arbusto, tres hierbas, dos trepadoras y una parásita (Cuadro 3). Aparte de estas, se colectaron ejemplares de plantas que según los especialistas pertenecen a dos especies nuevas para la zona de estudio: una trepadora del género Marsdenia R. Br. (Apocynaceae) y un árbol de Spondias L. (Anacardiaceae). Estas se anotan en el listado como "sp. nov." hasta que se publiquen sus descripciones.

En cuanto a la forma biológica predominan las plantas herbáceas terrestres $(\mathrm{H})$ con 121 taxones (34.3\%) y le siguen las especies arbóreas (A) con 109 (31\%). Las plan-

Cuadro 1: Composición de la flora del bosque tropical caducifolio (BTC) de la microcuenca La Salada, Colima, México.

\begin{tabular}{|c|c|c|c|c|c|}
\hline Grupo taxonómico & Familias & Géneros & Especies & Taxones infraespecíficos & Taxones total \\
\hline \multicolumn{6}{|l|}{ PTERIDOFITAS } \\
\hline Lycopodiopsida & 1 & 1 & 2 & & 2 \\
\hline Polypodiopsida & 3 & 8 & 9 & 2 & 11 \\
\hline \multicolumn{6}{|l|}{ ANGIOSPERMAS } \\
\hline Magnólidas & 4 & 4 & 6 & & 6 \\
\hline Monocotiledóneas & 10 & 27 & 42 & 1 & 42 \\
\hline Eudicotiledóneas & 57 & 193 & 293 & 14 & 307 \\
\hline Total & 75 & 233 & 352 & 16 & 368 \\
\hline
\end{tabular}

Cuadro 2: Familias y géneros con mayor riqueza de especies de la zona tropical yesosa y calcárea de la microcuenca La Salada, Colima, México.

\begin{tabular}{lccc}
\hline Familias & Número de Géneros/Especies & Género (Familia) & Número Taxones \\
\hline Fabaceae & $38 / 58$ & Euphorbia L. (Euphorbiaceae) & 13 \\
Euphorbiaceae & $10 / 31$ & Bursera Jacq. ex L. (Burseraceae) & 8 \\
Asteraceae & $20 / 25$ & Ipomoea L. (Convolvulaceae) & 7 \\
Malvaceae & $12 / 20$ & Tillandsia L. (Bromeliaceae) & 6 \\
Poaceae & $11 / 16$ & Croton L. (Euphorbiaceae) & 5 \\
Convolvulaceae & $6 / 16$ & & 39 \\
\hline Total & $\mathbf{9 7 / 1 6 6}$ & Total & 39 \\
\hline
\end{tabular}



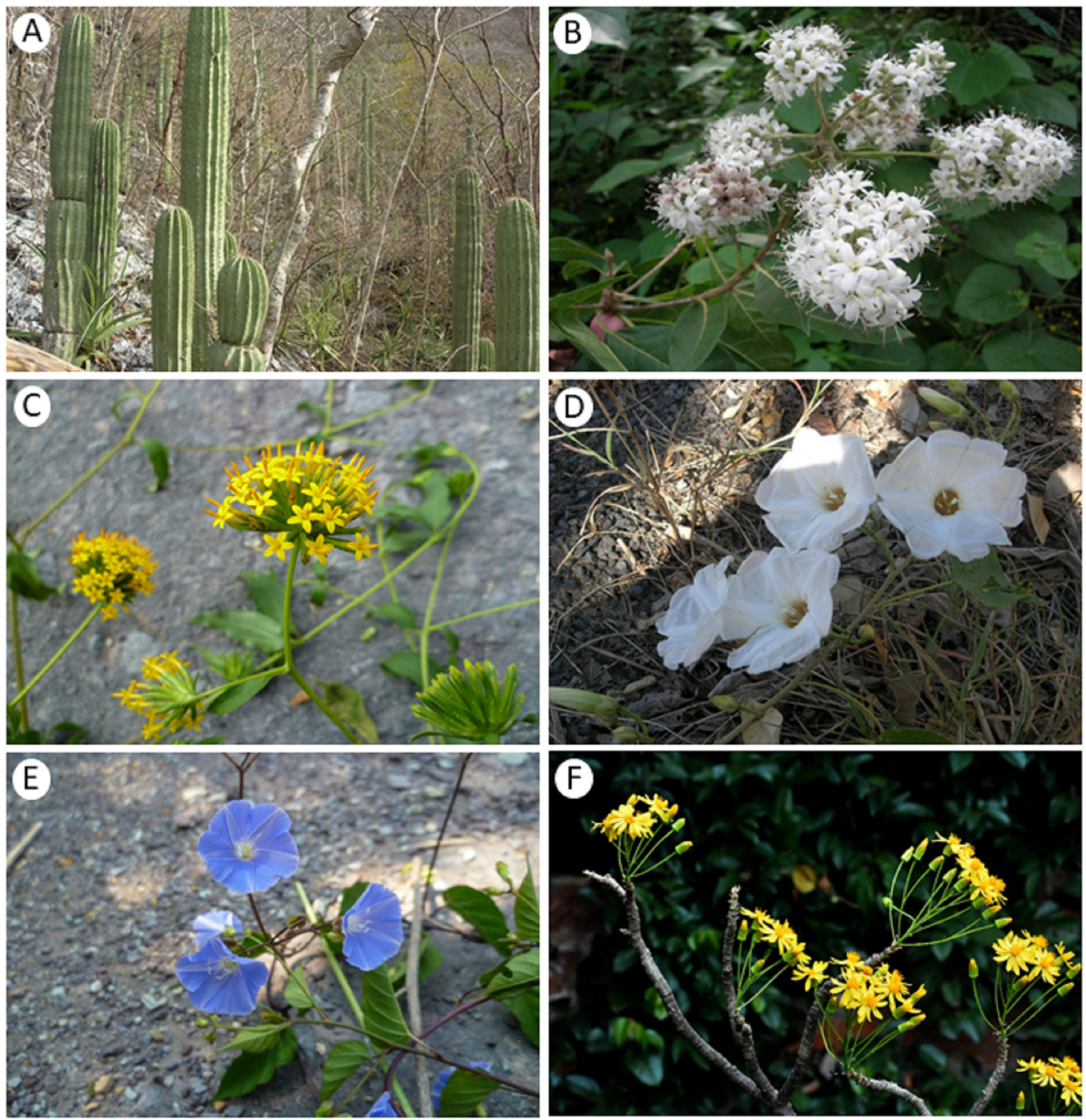

Figura 3: Algunas especies representativas del bosque tropical caducifolio de Colima, México. A. Cephalocereus nudus E.Y. Dawson; B. Cordia alliodora (Ruiz \& Pav.) Oken; C. Flaveria robusta Rose; D. Jacquemontia nelsonii House; E. Jacquemontia pentanthos (Jacq.) G. Don; F. Pittocaulon filare (McVaugh) H. Rob. \& Brettell. Fotografías de M. Harker (A-E) y de J. Aragón (F).

tas trepadoras ( $\mathrm{T}$ ) constan de 58 y las arbustivas (a) con 57 (16.4 y $16 \%$ respectivamente), mientras que solo $2 \%$ incluye a especies con hábito epífito (seis) y parásitas (dos).

En el Cuadro 4 se listan 40 colectores que han contribuido al conocimiento florístico del área de estudio. Cabe destacar el aporte de Rogers McVaugh, ya que se encontró a partir de la revisión bibliográfica y de sus libros de campo, que él colectó 199 números en total (incluyendo los colectados en compañía de Walter Koelz, que constituye una seriación diferente), que corresponden a 151 taxones (Apén- 
Cuadro 3: Taxones presentes en el área de estudio no registrados previamente en la literatura para el estado de Colima, México. Forma biológica: árbol $(\mathrm{A})$, hierba $(\mathrm{H})$, parásita $(\mathrm{P})$ y trepadora $(\mathrm{T})$.

\begin{tabular}{llc}
\hline \multicolumn{1}{c}{ Familia } & \multicolumn{1}{c}{ Taxones } & Forma biológica \\
\hline Acanthaceae & Ruellia longepetiolata (Oerst.) Hemsl. & $\mathrm{H}$ \\
Anacardiaceae & Spondias sp. nov. & $\mathrm{A}$ \\
Apiaceae & Prionosciadium acuminatum B.L. Rob. & $\mathrm{H}$ \\
Apocynaceae & Marsdenia sp. nov. & $\mathrm{T}$ \\
Asteraceae & Bidens reptans var. urbanii (Greenm.) O.E. Schulz & $\mathrm{H}$ \\
Convolvulaceae & Cuscuta macvaughii Yunck. & $\mathrm{P}$ \\
& Jacquemontia polyantha (Schltdl. \& Cham.) Hallier f. & $\mathrm{T}$ \\
Fabaceae & Coursetia oaxacensis M. Sousa \& Rudd & $\mathrm{A}$ \\
Nyctaginaceae & Grajalesia fasciculata (Standl.) Miranda & $\mathrm{A}$ \\
Rubiaceae & Guettarda filipes Standl. & $\mathrm{A}$ \\
Vitaceae & Vitis cinerea (Engelm.) Millardet & $\mathrm{T}$ \\
\hline
\end{tabular}

Cuadro 4: Colectores de la zona tropical yesosa y calcárea de Colima, México.

\begin{tabular}{cl}
\hline Abreviación & Nombre del colector \\
\hline AD & Alfonso Delgado Salinas \\
AFC & Alfredo Frías Castro \\
AG & Alwyn Gentry \\
ARC & Aarón Rodríguez Contreras \\
AS & Andrew Sanders \\
ASM & José Arturo Solís Magallanes \\
AVZ & Ana Valenzuela Zapata \\
CF & Charles Feddema \\
CGM & Cecilia García M. \\
DL & David H. Lorence \\
ECG & Eleazar Carranza González \\
EL & Emily Lott \\
FHM & Federico Halbinger Mosig \\
FMG & Faustino Miranda González \\
FSM & Francisco Javier Santana Michel \\
GW & Grady Webster \\
GML & Guadalupe Munguía Lino \\
HI & Hugh Iltis \\
JB & James A. Bauml \\
JD & Jennie Van Akkeren Dieterle \\
\hline
\end{tabular}

\begin{tabular}{cl}
\hline Abreviación & Nombre del colector \\
\hline JM & John Thomas Mickel \\
JPR & Jorge Alberto Pérez de la Rosa \\
JR & Jerzy Rzedowski \\
JRR & John Raymond Reeder \\
K & Walter Koelz \\
LHL & Leticia Hernández López \\
McV & Rogers McVaugh \\
MH & Mollie Harker \\
MPA & Manuel Peinado Arrellanes \\
PCR & Pablo Carrillo Reyes \\
PZT & Pilar Zamora Tavares \\
RA & Reino Alava \\
RC & Robert Cruden \\
RCG & Ramón Cuevas Guzmán \\
RRD & Raymundo Ramírez Delgadillo \\
SK & Stephen D. Koch \\
SZR & Sergio Zamudio Ruíz \\
TD & Thomas F. Daniel \\
TS & Tod Stuessy \\
WA & William Anderson \\
\hline & \\
\hline
\end{tabular}

dice 1). Mientras que en el presente trabajo corroboramos 82 de los taxa registrados por McVaugh, 69 solo se conocen a partir de los ejemplares colectados por él. Por otra parte, se adicionan 203 especies no documentados previamente para la zona.
Es importante notar que 43 (12\%) de los taxones presentes en la microcuenca La Salada se registran en la literatura como característicos de suelos yesosos y calcáreos, aunque no siempre se habla de exclusividad a esos tipos de sustrato. 
Otro aspecto sobresaliente del sitio es la localidad tipo de un género (Colima (Ravenna) Aarón Rodr. \& OrtizCatedral, Iridaceae) y de 23 especies, algunas de ellas además tienen distribución reducida (Cuadro 3).

En cuanto a la distribución geográfica de la flora, 39\% (144) de las especies registradas son endémicas de México (Apéndice 1). Sin embargo, la mitad de estas ocupan una superficie amplia en la entidad y el restante $50 \%$ (72) tienen distribución reducida. Por ejemplo, 34 especies se encuentran solo en la vertiente del Pacífico (de Sinaloa a Oaxaca), 27 ocupan el occidente de México (Nayarit, Jalisco, Colima y Michoacán) y de estas, dos especies, Hechtia laevis L.B. Sm. y Agave pablocarrilloi A. Vázquez, MuñizCastro \& Padilla-Lepe, son endémicas estrictas del estado de Colima (el Cuadro 5 muestra ejemplos de estas últimas). Las restantes 11 especies cubren parte de la vertiente Pací-

Cuadro 5: Especies con localidad tipo en la zona tropical yesosa y calcárea de Colima y que tienen distribución reducida en el país. Los estados se abrevian como: Colima (Col), Guerrero (Gro), Jalisco (Jal), Michoacán (Mich), Oaxaca (Oax).

\begin{tabular}{|c|c|c|c|}
\hline Familia & Taxones & Localidad Tipo & Distribución \\
\hline \multicolumn{4}{|l|}{ Polypodiopsida } \\
\hline Anemiaceae & Anemia colimensis Mickel & $\mathrm{x}$ & Col, Gro, Oax \\
\hline \multicolumn{4}{|l|}{ Magnólidas } \\
\hline Aristolochiaceae & Aristolochia colimensis Santana Mich. & $\mathrm{x}$ & Col, Jal \\
\hline Piperaceae & Piper abalienatum Trel. & $\mathrm{x}$ & Col, Jal, Mich \\
\hline \multicolumn{4}{|c|}{ Monocotiledóneas } \\
\hline \multirow[t]{2}{*}{ Asparagaceae } & Agave pablocarrilloi A. Vázquez, Muñiz-Castro \& Padilla-Lepe & $\mathrm{x}$ & Col \\
\hline & Polianthes howardii Verh.-Will. & & Col, Jal \\
\hline \multirow[t]{3}{*}{ Bromeliaceae } & Hechtia laevis L.B. Sm. & $\mathrm{x}$ & Col \\
\hline & Pitcairnia colimensis L.B. Sm. & $\mathrm{x}$ & Col, Mich \\
\hline & Tillandsia diguetii Mez \& Rol.-Goss. & & Col, Jal \\
\hline Dioscoreaceae & Dioscorea oreodoxa B.G. Schub. & $\mathrm{X}$ & Col, Jal \\
\hline Iridaceae & Colima convoluta (Ravenna) Aarón Rodr. \& Ortiz-Catedral & $\mathrm{x}$ & Col, Gro, Jal \\
\hline Poaceae & Bouteloua elata Reeder \& C. Reeder & $\mathrm{X}$ & Pacífico \\
\hline \multicolumn{4}{|l|}{ Eudicotiledóneas } \\
\hline Acanthaceae & Tetramerium mcvaughii T.F. Daniel & $\mathrm{X}$ & Col, Jal \\
\hline Amaranthaceae & Celosia moquini Guill. & & Col, Mich \\
\hline \multirow[t]{5}{*}{ Asteraceae } & Lepidaploa koelzii (McVaugh) H. Rob. & $\mathrm{x}$ & Col, Jal \\
\hline & Melampodium nutans Stuessy & $\mathrm{x}$ & Pacífico \\
\hline & Montanoa laskowskii McVaugh & $\mathrm{X}$ & Col, Jal \\
\hline & Pittocaulon filare (McVaugh) H. Rob \& Brettell & $\mathrm{X}$ & Pacífico y centro \\
\hline & Verbesina mickelii McVaugh & $\mathrm{x}$ & Col, Jal \\
\hline Fabaceae & Bauhinia gypsicola McVaugh & $\mathrm{x}$ & Col, Jal, Mich \\
\hline Lentibulariaceae & Pinguicula colimensis McVaugh \& Mickel & $x$ & Col, Mich \\
\hline \multirow[t]{3}{*}{ Malpighiaceae } & Gaudichaudia mcvaughii W.R. Anderson & $x$ & Pacífico \\
\hline & Malpighia novogaliciana W.R. Anderson & $x$ & Pacífico \\
\hline & Malpighia rzedowskii W.R. Anderson & $x$ & Pacífico \\
\hline \multirow[t]{2}{*}{ Malvaceae } & Abutilon bastardioides Baker f. ex Rose & $x$ & Col, Jal \\
\hline & Waltheria bicolor J.G. Saunders & $x$ & Col, Jal \\
\hline Orobanchaceae & Lamourouxia colimae W.R. Ernst \& Baad & $\mathrm{x}$ & Col, Jal \\
\hline Phyllanthaceae & Phyllanthus gypsicola McVaugh & & Col, Jal \\
\hline
\end{tabular}


fica, pero extienden su distribución hacia algunos estados del centro del país como al Estado de México y Zacatecas.

Los resultados del cálculo del índice de diversidad taxonómica (IDT) para La Salada (330.35 especies/In área) y para otros sitios representativos con dominancia de BTC en México se muestran en el Cuadro 6.

Se pueden adicionar a estos datos los registros obtenidos a partir de bases de datos digitales sobre colectas realizadas en el BTC en suelos yesosos del municipio Coquimatlán, Colima (Apéndice 2). Tales registros representan 75 taxones (incluyendo dos de categoría infraespecífica) en 61 géneros y 36 familias. Algunos de estos se comparten con La Salada, área muestreada por los autores (Apéndice 1), por lo que el aporte final de los registros de Coquimatlán consta de 40 taxones, 15 géneros y tres familias. Se integra así, el gran total de la flora del BTC en suelos yesosos y calcáreos del estado de Colima registrado en este trabajo es de 408 taxones, 248 géneros y 78 familias.

\section{Discusión}

En el área de estudio destaca la familia Fabaceae por su diversidad de especies, lo cual coincide con lo encontrado por otros autores en bosques tropicales caducifolios (Rzedowski, 1978, 1998; Gentry, 1995; Lott y Atkinson, 2010;
Méndez-Toribio et al., 2016). No obstante, Cuevas-Guzmán et al. (1998) registran para el BTC de la Sierra de Manantlán (Jalisco y Colima) a Asteraceae, Fabaceae, Poaceae y Euphorbiaceae como las familias de mayor riqueza. Esto se debe en parte a que en porciones de esa área el BTC alcanza elevaciones de hasta 2000 m, lo cual contrasta con el límite altitudinal de $1600 \mathrm{~m}$ propuesto en otros estudios (Gentry, 1995). Otras familias diversas en el área son Euphorbiaceae, Malvaceae, Poaceae y Convolvulaceae, que coinciden en parte con lo registrado por Lott (1993) y Lott y Atkinson (2010) para la vertiente del Pacífico. Estos autores sugieren que el grado de aridez en este ecosistema influye en gran medida en la composición al nivel de familia. Euphorbia, Ipomoea y Tillandsia son tres de los géneros reconocidos aquí como los más diversos y también se registran así para Chamela, Jalisco (Gentry, 1995). Euphorbia y Tillandsia destacan entre los tres géneros más diversos de la flora de México (Villaseñor, 2016).

Una aportación del presente trabajo es que se amplía la distribución de 11 taxones (diez especies y una variedad) que no se tenían registrados para Colima (Cuadro 3). Algunos de ellas tienen distribución amplia en el país (Villaseñor, 2016), por lo que no es sorprendente que se hayan encontrado también en Colima. No obstante, dos

Cuadro 6: Comparación de la riqueza florística del bosque tropical caducifolio (BTC) de Colima con otras áreas cuya vegetación dominante es el BTC y utilizando el índice de diversidad taxonómica (IDT). InA=logaritmo natural del área en km²; F=familias; G=géneros; ND=no disponible. Los tipos de vegetación con base en Rzedowski (1978): BG=bosque de galería; BTS=bosque tropical subcaducifolio; VH=vegetación halófila.

\begin{tabular}{|c|c|c|c|c|c|c|c|c|c|}
\hline Fuente & Localidad & $\begin{array}{l}\text { Área } \\
\left(\mathrm{km}^{2}\right)\end{array}$ & $\begin{array}{c}\text { No. de } \\
\text { exploraciones }\end{array}$ & $\begin{array}{l}\text { Elevación } \\
\text { m s.n.m. }\end{array}$ & $\begin{array}{l}\text { Tipos de } \\
\text { vegetación }\end{array}$ & $\mathbf{F}$ & G & Taxa & $\begin{array}{c}\text { IDT } \\
\text { (Sp/InA) }\end{array}$ \\
\hline León de la Luz et al., 2012 & $\begin{array}{l}\text { Península de Baja } \\
\text { California }\end{array}$ & 3325 & ND & $200-1,200$ & BTC & 101 & 360 & 645 & 79.54 \\
\hline Pérez-García et al., 2010 & $\begin{array}{l}\text { Región de Nizanda, } \\
\text { Oaxaca }\end{array}$ & 80 & ND & $100-750$ & BTC & 82 & 283 & 424 & 96.76 \\
\hline $\begin{array}{l}\text { Zepeda-Gómez y Velázquez- } \\
\text { Montes, } 1999\end{array}$ & $\begin{array}{l}\text { Sierra de Nanchititla, } \\
\text { Estado de México }\end{array}$ & 13.2 & 15 & $600-1400$ & BTC & 82 & 208 & 266 & 103.09 \\
\hline Ramírez-Díaz, 2016 & $\begin{array}{c}\text { Subcuenca Cuixtla, } \\
\text { Jalisco-Zacatecas }\end{array}$ & 123 & 50 & $810-1770$ & BTC, BG & 108 & 373 & 610 & 126.76 \\
\hline Lott, 1993 & $\begin{array}{l}\text { Bahía de Chamela, } \\
\text { Jalisco }\end{array}$ & 350 & ND & $0-500$ & BTC, BTS, VH & 124 & 544 & 1,120 & 191.19 \\
\hline Cuevas-Guzmán et al., 1998 & $\begin{array}{l}\text { Sierra de Manantlán, } \\
\text { Jalisco-Colima }\end{array}$ & 127 & ND & $400-2000$ & BTC & 111 & 531 & 1,067 & 220.26 \\
\hline Este trabajo & La Salada, Colima & 2.92 & 9 & $370-600$ & BTC & 75 & 233 & 368 & 330.35 \\
\hline
\end{tabular}


de las especies aún no han sido descritas ya que son nuevas para la ciencia, por lo que constituyen aportaciones a la flora tanto estatal como nacional. Los listados florísticos publicados recientemente facilitan una evaluación o comparación de la riqueza florística: Ulloa-Ulloa et al. (2017) para las Américas y Villaseñor (2016) para México. Nuestros resultados confirman lo planteado por varios autores donde se enfatiza que la actualización de los listados florísticos amplía el conocimiento de la biota (Villaseñor, 2016), en particular en áreas poco estudiadas y con bosques tropicales caducifolios donde existe un alto recambio de especies entre distintos sitios (Trejo y Dirzo, 2002). En el área hemos observado que la presencia de algunas especies depende de la exposición de la ladera (orientación). Tal es el caso de Begonia monophylla Pav. ex A. DC. y Pinguicula colimensis McVaugh \& Mickel que solo habitan en laderas de exposición norte. Otras especies crecen en los márgenes de charcas (Eustoma exaltatum (L.) Salisb. ex G. Don) y en los arroyos al lado de la carretera o entre las colinas, como Acrostichum danaeifolium Langsd. \& Fisch.

EI BTC favorece el desarrollo de diferentes formas biológicas (Gentry, 1995; Cuevas-Guzmán et al., 1998). En el área destacan las hierbas terrestres, y las familias con mayor número de especies son Poaceae, Euphorbiaceae y Asteraceae. Las cuatro familias que aportan más a los árboles son Fabaceae (38 taxa), Burseraceae (ocho), Anacardiaceae (cinco) y Rhamnaceae (cuatro). Eso coincide parcialmente con lo encontrado por Moreno-Gómez et al. (2016), donde Fabaceae (23), Anacardiaceae, Malvaceae, Moraceae (cinco c/u) y Boraginaceae (cuatro) son las familias con más árboles. Es importante destacar que la zona de estudio cubre solo 292 ha y se registran 109 especies de árboles, mientras que Moreno-Gómez et al. (2016) listan 65 para la microcuenca La Salada que ocupa una superficie 38 veces mayor (7850 ha). Sin embargo, en ese estudio se seleccionaron las especies arbóreas más representativas y probablemente a eso se deba el contraste en el número de especies. La proporción de riqueza de especies de herbáceas aquí mencionada (34.3\%) contrasta con $43 \%$ registrado por Cuevas-Guzmán et al. (1998) para el BTC de la Sierra de Manantlán, donde además el hábito arbustivo ocupa la segunda posición después el arbóreo. Lo anterior indica que la estructura del BTC es diferente en los dos sitios.
El número de colectores botánicos (40) que han aportado al conocimiento de la flora del área de estudio seguramente se incrementará con la revisión de otros herbarios no contemplados en este trabajo. Cabe mencionar que, de los colectores registrados aquí, la mayoría han explorado de forma esporádica (ver Apéndices 1 y 2). Se enfatiza el trabajo realizado por McVaugh y el hecho de que 70 de los taxones reportados por él no se hayan logrado recolectar en esta aportación, lo cual sugiere que se requiere mayor esfuerzo de colecta o que haya ocurrido un proceso de extinción local. Por otra parte, es interesante notar que 81 de las especies registradas por él se hayan recolectado, es decir, permanecen en el área después de casi 50 años.

La superficie ocupada por suelos yesosos y calcáreos en Colima es reducida si se compara con la registrada en otras regiones de México, como el desierto Chihuahuense (que en su porción mexicana incluye parcialmente a los estados de Chihuahua, Coahuila, Durango, Nuevo León, San Luis Potosí, Tamaulipas y Zacatecas) y la Península de Yucatán. Estos suelos presentan características que los convierten en hábitat hostil en extremo para muchas plantas (Moore y Jansen, 2007). Los suelos derivados de yeso presentan propiedades químicas adversas como la falta de nutrientes en el suelo (Meyer et al., 1992) y la alta concentración de iones de sulfato que pueden llegar a ser tóxicos para las plantas (Ruiz et al., 2003). Por otra parte, entre sus peculiaridades físicas, que también ocasionan elevado estrés, se mencionan la existencia de una costra endurecida en la superficie del suelo que puede limitar el establecimiento de plántulas (Meyer, 1986), y la alta retención de agua por los sulfatos, que provoca una aridez fisiológica al dificultar la disponibilidad de agua para las raíces de las plantas (Rzedowski, 1978; Turner y Powell, 1979). Además, la falta de plasticidad vuelve al suelo muy inestable (Bridges y Burnham, 1980). Así mismo, en algunas áreas, la penetración de las raíces se ve limitada por la falta de porosidad en el suelo (Palacio et al., 2007). No obstante, los suelos yesosos pueden dar origen a especies endémicas y raras (Meyer, 1986; Palacio et al., 2007). Estos mismos autores plantean que se trata de especies tolerantes a condiciones de estrés, pero que también pueden crecer en otros hábitats ("gipsovagas") o bien, de aquellas especializadas a estos hábitats ad- 
versos ("gipsófilas") constituyendo endemismos edáficos. En este sentido, en el área de estudio, se han descrito algunas especies cuyo epíteto especifico hace referencia a estos sustratos y restringen su distribución al occidente de México, por ejemplo: Bauhinia gypsicola McVaugh y Phyllanthus gypsicola McVaugh hacen alusión a los suelos derivados de yeso, mientras que Euphorbia calcarata (Schltdl.) V.W. Steinm. señala suelos calcáreos. Sin embargo, se requieren otros estudios para determinar si son especies exclusivas de esos sustratos.

Para el territorio de México no se encontraron estudios previos de BTC como vegetación dominante y exclusiva de una zona yesosa; sin embargo, para la zona yesosa-calcárea de meseta de Zoh-Laguna en Calakmul, Campeche, hay algunos estudios en los que se reporta BTC como uno de los diez tipos de vegetación presentes (Martínez et al., 2001; Martínez y Galindo-Leal, 2002), pero en estos trabajos no se hace un análisis por separado de la riqueza del BTC, ni por tipo de sustrato. Además, el BTC en Zoh-Laguna representa solo una proporción menor entre una matriz mayor de selvas medianas subperennifolias, ya que García-Gil et al. (2001) reportan una cobertura de BTC de solo $0.34 \%$ del área de la Reserva de Calakmul. Los estudios florísticos en suelos yesosos se han concentrado más bien en zonas templadas áridas y semiáridas donde la vegetación dominante es el matorral xerófilo (Johnston, 1941; Rzedowski, 1955; Ochoterena et al., 2020). Sin embargo, al igual que en el área de La Salada, Colima, las comunidades de BTC de Calakmul también contienen endemismos muy localizados en sus afloramientos yesosos, como son los de las especies Holographis websteri T.F. Daniel y Lantana dwyeriana Moldenke (Martínez y Galindo-Leal, 2002). Diversos autores han enfatizado el riesgo que enfrentan los suelos yesosos y calcáreos. Según Román-Guzmán et al. (2016), la vegetación con estos tipos de suelos ha disminuido considerablemente en Colima. Así mismo, Ramírez-Delgadillo et al. (2016) reiteran el peligro que representa la industria de la cal y el yeso, pues amenaza la diversidad florística en áreas con bosque tropical caducifolio de Colima. En la zona de estudio se ha observado que la explotación del suelo para la fabricación comercial de cal ha ido en aumento y esto podría significar la extirpación de algunas especies de distribución restringida.
Es sobresaliente el hecho de que en un área tan reducida (292 ha o $2.92 \mathrm{~km}^{2}$ ) se encuentren 144 especies endémicas de México. Eso representa $8.4 \%$ de las endémicas que Villaseñor (2016) registra para todo el estado de Colima. Lott y Atkinson (2010) plantean que el endemismo de especies en el BTC es alto (60\%), pero reducido al nivel de géneros. Es interesante que en el área se registra el género Colima (Rodríguez y Ortiz-Catedral, 2003) que se restringe a Jalisco, Colima y Guerrero. Destacan dos especies endémicas estrictas y 15 especies cuasi endémicas, es decir conocidas solo de Colima y algún estado colindante. La presencia de los suelos derivados de yeso y de roca caliza, con sus particularidades ya mencionadas, además del clima cálido, podrían explicar la notable diversidad y endemismo encontrado en el área. Algo similar se ha observado en estudios florísticos del desierto Chihuahuense (Villarreal-Quintanilla et al., 2017) y de Coahuila (Villarreal y Encina, 2005), aunque en ellos los tipos de vegetación con mayor endemismo son el matorral xerófilo y el matorral submontano. Otros trabajos han documentado cómo las condiciones adversas de los suelos yesosos limitan el crecimiento de especies arbóreas, en consecuencia, en la vegetación dominan las formas herbáceas y arbustivas (Rivas-Martínez y Costa, 1970). Sin embargo, en nuestra área de estudio la forma arbórea casi iguala en proporción a las hierbas y predomina sobre las arbustivas. Por otra parte, es interesante que el número de taxones con hábito trepador casi empate con el arbustivo.

El valor calculado del índice de diversidad taxonómica (IDT) del área de estudio de Colima (330.35 especies/In área) es mucho mayor al de otras áreas de BTC de México (Lott, 1993; Cuevas-Guzmán et al., 1998; Zepeda-Gómez y Velázquez-Montes, 1999; Pérez-García et al., 2010; León de la Luz et al., 2012; Ramírez-Díaz, 2016) (Cuadro 6). Los cálculos del índice de diversidad taxonómica (IDT) se hicieron sobre las unidades de vegetación de BTC en la mayoría de los casos reportados en el Cuadro 6. Incluso en los dos que se reporta presencia también de bosque de galería (BG) (Zepeda-Gómez y Velázquez-Montes, 1999; RamírezDíaz, 2016), esta vegetación es marginal en esos estudios, con una representación en área muy baja. En el estudio de Lott (1993), además del BTC también se incluyen los tipos de vegetación de bosque tropical subcaducifolio (BTS) y 
vegetación halófila $(\mathrm{VH})$, pero aún en este estudio la mayor representación es de la unidad vegetal de BTC. Por ello consideramos que estos cálculos de diversidad taxonómica son representativos de las unidades vegetales de BTC de todos estos estudios. Hay que considerar que en el cálculo de este índice se tomaron en cuenta registros históricos de McVaugh, de los cuales 70 taxones no se encontraron en colectas recientes. Sin embargo, si se eliminan esos 70 registros históricos del cálculo del IDT, el resultado sigue siendo alto, con 265.03 especies/In área. Esta alta diversidad es mayor a la esperada de acuerdo al tipo de suelo litosol yesoso y calcáreo de la zona de estudio, sustratos someros que no retienen la humedad superficial, pero que propician micro-hábitats que pueden funcionar como litorefugios que promueven diversificación y una alta riqueza de endemismos, como se ha documentado para otros BTC en suelos calcáreos (de Aguiar-Campos et al., 2020).

EI BTC de México y Centroamérica se ubica entre los ecosistemas de mayor prioridad para la conservación de biodiversidad (Ceballos y García, 1995) y a escala regional los BTC de Jalisco y la Cuenca del Balsas tienen la más alta prioridad por su elevada diversidad y endemismo tanto de flora como de fauna (Lott y Atkinson, 2010). En Colima, este ecosistema tiene poca representación en el sistema de áreas naturales protegidas continentales, dado que solamente una pequeña porción de la Reserva de la Biosfera Sierra de Manantlán corresponde a Colima y otro bajo porcentaje del Área de Protección Forestal y Refugio de Fauna Silvestre El Jabalí incluye este tipo de vegetación (Cuevas-Guzmán et al., 1998; Sáyago-Lorenzana et al., 2016). Fomentar los inventarios biológicos en áreas poco conocidas, en particular las de BTC en sustrato yesoso, así como la realización de estudios poblacionales de especies prioritarias, ayudarán a fundamentar esquemas de conservación de biodiversidad con un enfoque integral, de modo que la permanencia del BTC en la entidad sea una realidad.

\section{Contribución de autores}

MH realizó trabajo de campo, determinación de exsiccata, consulta del Herbario MICH y redacción del artículo. LHL llevó a cabo trabajo de campo, análisis de los datos y redacción del artículo. MAMC efectuó análisis de los datos y redacción del manuscrito. Todos los autores revisaron y retroalimentaron el manuscrito hasta su versión final.

\section{Financiamiento}

El Programa Integral para el Fortalecimiento Institucional (PIFI) de la SEP, a través de la Universidad de Guadalajara, financió la visita de una de las autoras al Herbario de la Universidad de Michigan (MICH) en los Estados Unidos. EI trabajo de campo fue financiado con recursos de $\mathrm{MH}$ y LHL.

\section{Agradecimientos}

Agradecemos por su ayuda con algunas determinaciones a J. Jacqueline Reynoso D. (Fabaceae), Raymundo Ramírez D. (varias familias), José Luis Villalpando P. y Eduardo Ruíz S. (Poaceae), Jesús G. González G. (Lamiaceae), Pablo Carrillo R., Karina Machuca M., Eloy Padilla V., Ramón Cuevas G., Francisco J. Santana M., Hilda J. Arreola N., Christine Dudding, Manuel Peinado A., Laura Rodríguez y Pilar Zamora T. ayudaron en el campo. Víctor Quintero F., Claudia J. Ramírez D., y Sofía Monroy S. apoyaron con determinaciones taxonómicas de varias familias y en la base de datos. Apreciamos al curador del herbario $\mathrm{MICH}$ por facilitar el acceso a los libros de colecta de Rogers McVaugh y sus ejemplares depositados en éste. Se agradece a Darío Figueroa G. por la edición de las imágenes y arreglo de las láminas y a Juvenal Aragón P. por las fotos de Pittocaulon filare. Saúl Moreno Gómez proporcionó información digital sobre suelos de la zona. Los mapas y la determinación de las regiones biogeográficas que ocupan el área fueron elaborados por G. Munguía L. del Laboratorio de Biogeografía de LANIVEG, en la Universidad de Guadalajara. A Aarón Rodríguez por proporcionar datos de sus colectas en la zona y por la revisión y sugerencias al manuscrito. Nuestro agradecimiento a los revisores anónimos y al equipo editorial por su retroalimentación para la mejora del documento.

\section{Literatura citada}

APG IV. 2016. An update of the Angiosperm Phylogeny Group classification for the orders and families of flowering plants: APG IV. Botanical Journal of the Linnean Society 181(1): 1-20. DOI: https://doi.org/10.1111/boj.12385

Arévalo, G. G., M. G. Rodríguez-Camarillo y A. G. Miranda M. 2016. Descripción de los principales tipos de vegetación. 
In: Comisión Nacional para el Conocimiento y Uso de la Biodiversidad (ed.). La Biodiversidad en Colima. Estudio de Estado-Comisión Nacional para el Conocimiento y Uso de la Biodiversidad (CONABIO). Cd. Mx., México. Pp. 116-131.

Báez-Montes, O. 2016a. Resumen ejecutivo de Medio físico. In: Comisión Nacional para el Conocimiento y Uso de la Biodiversidad (ed.). La Biodiversidad en Colima. Estudio de Estado-Comisión Nacional para el Conocimiento y Uso de la Biodiversidad (CONABIO). Cd. Mx., México. Pp. 23-24.

Báez-Montes, O. 2016b. Resumen ejecutivo de Diversidad de especies "Hongos y plantas". In: Comisión Nacional para el Conocimiento y Uso de la Biodiversidad (ed.). La Biodiversidad en Colima. Estudio de Estado-Comisión Nacional para el Conocimiento y Uso de la Biodiversidad (CONABIO). Cd. Mx., México. Pp. 175-176.

Bezaury C., J. 2010. Las selvas secas del Pacífico mexicano en el contexto mundial. In: Ceballos, G., L. Martínez, A. García, E. Espinoza, J. Bezaury y R. Dirzo (eds.). Diversidad, amenazas y áreas prioritarias para la conservación de las selvas secas del Pacífico de México. Fondo de Cultura Económica, Comisión Nacional para el Conocimiento y Uso de la Biodiversidad (CONABIO). Cd. Mx., México. Pp. 21-40.

Boyle, B., N. Hopkins, Z. Lu, J. A. Raygoza Garay, D. Mozzherin, T. Rees, N. Matasci, M. L. Narro, W. H Piel, S. J. Mckay, S. Lowry, C. Freeland, R. K. Peet y B. J. Enquist. 2013. The taxonomic name resolution service: an online tool for automated standardization of plant names. BMC Bioinformatics 14: 16. DOI: https://doi.org/10.1186/1471-2105-14-16

Bridges, E. M. y C. P. Burnham. 1980. Soils of the state of Bahrain. Journal of Soil Science 31(4): 689-707. DOI: https://doi. org/10.1111/j.1365-2389.1980.tb02115.x

Castro-Caro, E. 2016. Hidrología y clima. In: Comisión Nacional para el Conocimiento y Uso de la Biodiversidad (ed.). La Biodiversidad en Colima. Estudio de Estado-Comisión Nacional para el Conocimiento y Uso de la Biodiversidad (CONABIO). Cd. Mx., México. Pp. 44-50.

Ceballos, G. y A. García. 1995. Conserving Neotropical biodiversity: the role of dry forest in Western Mexico. Conservation Biology 9(6): 1349-1356.

Ceballos, G. y D. Valenzuela. 2010. Diversidad, ecología y conservación de los vertebrados de Latinoamérica. In: Ceballos, G., L. Martínez, A. García, E. Espinoza, J. Bezaury y R. Dirzo (eds.). Diversidad, amenazas y áreas prioritarias para la conservación de las selvas secas del Pacífico de México. Fondo de Cultura Económica, Comisión Nacional para el Conocimiento y Uso de la Biodiversidad (CONABIO). Cd. Mx., México. Pp. 93-118.

Ceballos, G., L. Martínez, A. García, E. Espinoza, J. Bezaury y R. Dirzo (eds.). 2010. Diversidad, amenazas y áreas prioritarias para la conservación de las selvas secas del Pacífico de México. Fondo de Cultura Económica, Comisión Nacional para el Conocimiento y Uso de la Biodiversidad (CONABIO). Cd. Mx., México. 594 pp.

Cervantes-Zamora, Y., S. L. Cornejo-Olguín, R. Lucero-Márquez, J. M. Espinoza-Rodríguez, E. Miranda-Víquez y E. Pineda Velásquez. 1990. Provincias fisiográficas de México. In: Atlas Nacional de México-Clasificación de Regiones Naturales de México II, Vol. II. Escala 1:4 000 000. Instituto de Geografía, Universidad Nacional Autónoma de México. Cd. Mx., México. http://www.conabio.gob.mx/informacion/ metadata/gis/renat4mgw.xml?_xsl=/db/metadata/xsl/ fgdc_html.xsl\&_indent=no (consultado agosto de 2019).

CONABIO. 2004. Mapa base del estado de Colima: Catálogo de metadatos geográficos. Comisión Nacional para el Conocimiento y Uso de la Biodiversidad (CONABIO). Cd. Mx., México. http://www.conabio.gob.mx/informacion/ metadata/gis/colaprgn.xml?_httpcache=yes\&_xsl=/ $\mathrm{db} /$ metadata/xsl/fgdc_html.xsl\&_indent=no\&as=.html (consultado agosto de 2020).

CONABIO. 2016. La Biodiversidad en Colima: Estudio de Estado. Comisión Nacional para el Conocimiento y Uso de la Biodiversidad (CONABIO). Cd. Mx., México. 766 pp.

CONABIO. 2019. Portal de Geoinformación: Sistema Nacional de Información sobre Biodiversidad. http://www.conabio.gob. mx/informacion/gis/ (consultado junio de 2019).

Cuevas-Guzmán, R., N. M. Núñez L., E. Sánchez R., L. Guzmán H. y F. J. Santana M. 1998. El bosque tropical caducifolio en la Reserva de la Biósfera Sierra de Manantlán, Jalisco-Colima, México. Boletín del Instituto de Botánica 5(1-3): 445-491.

Daniel, T. F. 1986. Systematics of Tetramerium (Acanthaceae). Systematic Botany Monographs 12: 1-134. DOI: https://doi. $\operatorname{org} / 10.2307 / 25027630$

de Aguiar-Campos, N., V. A. Maia, W. B. da Silva, C. R. de Souza y R. M. dos Santos. 2020. Can fine-scale habitats of limestone outcrops be considered litho-refugia for dry forest tree lineages? Biodiversity and Conservation 29(3): 1009-1026. 
DOI: https://doi.org/10.1007/s10531-019-01923-4

Dinerstein, E., D. M. Olson, D. J. Graham, A. L. Webster, S. A. Primm, M. P. Bookbinder y G. Ledec. 1995. A Conservation Assessment of the Terrestrial Ecoregions of Latin America and the Caribbean. World Wildlife Fund-The World Bank. Washington, D.C., EUA. 121 pp. DOI: https://doi. org/10.1596/0-8213-3295-3

DRYFLOR. 2016. Plant diversity patterns in neotropical dry forests and their conservation implications. Science 353(6306): 1383-1387. DOI: https://doi.org/10.1126/ science.aaf5080

Ernst, W. R. y M. F. Baad. 1970. Two new species of Lamourouxia (Scrophulariaceae) in Mexico. Madroño 20(7): 342-346.

García, A. 2010. Colima Centro, Colima, Jalisco y Michoacán. In: Ceballos, G., L. Martínez, A. García, E. Espinoza, J. Bezaury y R. Dirzo (eds.). Diversidad, amenazas y áreas prioritarias para la conservación de las selvas secas del Pacífico de México. Fondo de Cultura Económica, Comisión Nacional para el Conocimiento y Uso de la Biodiversidad (CONABIO). Cd. Mx., México. Pp. 458-460.

García-Gil, G., I. March-Mifsut y M. Á. Castillo-Santiago. 2001. Transformación de la vegetación por cambio de uso del suelo en la Reserva de la Biosfera Calakmul, Campeche. Investigaciones Geográficas, Boletín del Instituto de Geografía, Universidad Nacional Autónoma de México (UNAM) 46: 45-57.

GBIF. 2021. Global Biodiversity Information Facility. GBIF.org. GBIF Occurrence Download. Copenhague, Dinamarca. DOI: https://doi.org/10.15468/dl.qdja2r

Gentry, A. H. 1995. Diversity and floristic composition of Neotropical dry forests. In: Bullock, S. H., H. A. Mooney y E. Medina (eds.). Seasonally dry forests. Cambridge University Press. New York, EUA. Pp. 146-194.

Hinton, J. y G. S. Hinton. 1995. Checklist of Hinton's collections of the flora of south-central Nuevo Leon and adjacent Coahuila. Acta Botanica Mexicana 30: 41-112. DOI: https:// doi.org/10.21829/abm30.1995.732

IBUNAM. 2018. Herbario Nacional de México (MEXU), Plantas Vasculares. Portal de Datos Abiertos UNAM Colecciones Universitarias (en línea), Departamento de Botánica, Instituto de Biología, Universidad Nacional Autónoma de México (UNAM). Cd. Mx., México. http://datosabiertos. unam.mx (consultado junio de 2018).
IBUNAM. 2021. Herbario Nacional de México (MEXU), Plantas Vasculares. Portal de Datos Abiertos UNAM Colecciones Universitarias (en línea), Departamento de Botánica, Instituto de Biología, Universidad Nacional Autónoma de México (UNAM). Cd. Mx., México. http://datosabiertos. unam.mx (consultado enero de 2018).

INEGI. 2001. Conjunto de datos vectoriales Fisiográficos. Continuo Nacional, serie I. Provincias fisiográficas. Instituto Nacional de Estadística y Geografía. Aguascalientes, México. https://www.inegi.org.mx/app/biblioteca/ficha. html?upc=702825267575 (consultado octubre de 2020).

INEGI. 2013. Continuo de Elevaciones Mexicano 3.0 (CEM 3.0). Instituto Nacional de Estadística y Geografía. Aguascalientes, México. https://www.inegi.org.mx/app/ geo2/elevacionesmex/ (consultado diciembre de 2020).

INEGI. 2017. Conjunto de datos vectoriales de la Carta de Uso del Suelo y Vegetación. Escala 1:250 000, Serie VI (Conjunto Nacional). Instituto Nacional de Estadística y Geografía. Aguascalientes, México. https://www.inegi.org.mx/temas/ usosuelo/ (consultado enero de 2021).

Johnston, I. M. 1941. Gypsophily among Mexican desert plants. Journal of the Arnold Arboretum 22(2): 145-170.

León de la Luz, J. L., R. Domínguez-Cadena y A. Medel-Narváez. 2012. Florística de la selva baja caducifolia de la península de Baja California, México. Botanical Sciences 90: 143-162. DOI: https://doi.org/10.17129/botsci.480

Lot, A. y F. Chiang. 1986. Manual de Herbario. Administración, Manejo de Colecciones, Técnicas de Recolección y Preparación de Ejemplares Botánicos. Consejo Nacional de la Flora de México, A.C., Cd. Mx., México. 142 pp.

Lott, E. J. 1985. La Estación Biológica Chamela, Jalisco-Listados Florísticas de México III. Instituto de Biología, Universidad Nacional Autónoma de México. Cd. Mx., México. 46 pp.

Lott, E. J. 1993. Annotated checklist of the vascular flora of the Chamela bay region, Jalisco, México. Occasional papers of the California Academy of Sciences 148: 1-60.

Lott, E. J. у T. H. Atkinson. 2010. Diversidad florística. In: Ceballos, G., L. Martínez, A. García, E. Espinoza, J. Bezaury y R. Dirzo (eds.). Diversidad, amenazas y áreas prioritarias para la conservación de las selvas secas del Pacífico de México. Fondo de Cultura Económica, Comisión Nacional para el Conocimiento y Uso de la Biodiversidad (CONABIO). Cd. Mx., México. Pp. 63-76. 
Martínez, E. y C. Galindo-Leal. 2002. La vegetación de Calakmul, Campeche, México-clasificación, descripción y distribución. Boletín de la Sociedad Botánica de México 71: 7-32. DOI: https://doi.org/10.17129/botsci.1660

Martínez, E., M. Sousa y C. H. Ramos-Álvarez. 2001. Listados florísticos de México. XXII. Región de Calakmul, Campeche. Instituto de Biología, Universidad Nacional Autónoma de México. México, D.F., México. 55 pp.

McVaugh, R. 1972. Botanical Exploration in Nueva Galicia, México from 1790 to the present time. Contributions of the University of Michigan Herbarium 9(3): 205-357.

McVaugh, R. 1983. Flora Novo-Galiciana (Gramineae) XIV. University of Michigan Press. Ann Arbor, EUA. 436 pp.

McVaugh, R. 1984. Flora Novo-Galiciana (Compositae) XII. University of Michigan Press. Ann Arbor, EUA. 1157 pp.

McVaugh, R. 1985. Flora Novo-Galiciana (Orchidaceae) XVI. University of Michigan Press. Ann Arbor, EUA. 363 pp.

McVaugh, R. 1987. Flora Novo-Galiciana (Leguminosae) V. University of Michigan Press. Ann Arbor, EUA. 786 pp.

McVaugh, R. 1989. Flora Novo-Galiciana (Bromeliaceae to Dioscoreaceae) XV. University of Michigan Herbarium. Ann Arbor, EUA. 389 pp.

McVaugh, R. 1992. Flora Novo-Galiciana (Gymnosperms to Pteridophytes) XVII. University of Michigan Herbarium. Ann Arbor, EUA. 467 pp.

McVaugh, R. 1993. Flora Novo-Galiciana (Limnocharitaceae to Typhaceae) XIII. University of Michigan Herbarium. Ann Arbor, EUA. 480 pp.

McVaugh, R. 2001. Flora Novo-Galiciana (Ochnaceae to Loasaceae) III. University of Michigan Herbarium. Ann Arbor, EUA. $751 \mathrm{pp}$.

McVaugh, R. y J. T. Mickel. 1963. Notes on Pinguicula, sect. Orcheosanthus. Brittonia 15(2): 134-140. DOI: https://doi. $\operatorname{org} / 10.2307 / 2805399$

Méndez-Toribio, M., J. A. Meave, I.Zermeño-Hernández y G. IbarraManríquez. 2016. Effect of slope aspect and topographic position in environmental variables, disturbance and tree community attributes in the seasonal tropical dry forest. Journal of Vegetation Science 27: 1094-1103. DOI: https:// doi.org/10.1111/jvs.12455

Meyer, S. E. 1986. The ecology of gypsophile endemism in the Eastern Mojave desert. Ecology 67: 1303-1313. DOI: https:// doi.org/10.2307/1938686
Meyer, S. E., E. García-Moya y L. del C. Lagunes-Espinoza. 1992. Topographic and soil surface effects on gypsophile plant community patterns in central Mexico. Journal of Vegetation Science 3: 429-438. DOI: https://doi. org/10.1111/j.1654-1103.1992.tb00353.x

Miranda, F. y E. Hernández-X. 1963. Los tipos de vegetación de México y su clasificación. Chapingo. Boletín de la Sociedad Botánica de México 28: 29-179. DOI: https://doi. org/10.17129/botsci.1084

Moore, M. J. y R. K. Jansen. 2007. Origins and Biogeography of Gypsophily in the Chihuahuan Desert Plant Group Tiquilia Subg. Eddya (Boraginaceae). Systematic Botany 32(2): 392414. DOI: https://doi.org/10.1600/036364407781179680

Moreno-Gómez, S., R. Cuevas-Guzmán, N. M. Núñez-López y J. A. Solís-Magallanes. 2016. Guía de Árboles de la Selva Baja Caducifolia de la microcuenca La Salada, Colima-Colección Manantlán. Centro Universitario de la Costa Sur, Universidad de Guadalajara, Editorial Página Seis, S.A. de C.V., Zapopan, México. $87 \mathrm{pp}$.

Morrone, J. J. 2019. Regionalización biogeográfica y evolución biótica de México: encrucijada de la biodiversidad del Nuevo Mundo. Revista Mexicana de Biodiversidad 90: e902980. DOI: http://dx.doi.org/10.22201/ ib.20078706e.2019.90.2980

Morrone, J. J., T. Escalante y G. Rodríguez-Tapia. 2017. Mexican biogeographic provinces: Map and shapefiles. Zootaxa 4277: 277-279. DOI: https://doi.org/10.11646/zootaxa.4277.2.8

Ochoterena, H., H. Flores-Olvera, C. Gómez-Hinostrosa y M. J. Moore. 2020. Gypsum and plant species: a marvel of Cuatro Ciénegas and the Chihuahuan Desert. In: Mandujano, M., I. Pisanty y L. Eguiarte (eds.). Plant Diversity and Ecology in the Chihuahuan Desert. Cuatro Ciénegas Basin: An Endangered Hyperdiverse Oasis. Springer. Cham, Suiza. Pp. 129-165. DOI: https://doi.org/10.1007/978-3-030-449636_9

Olson, D. M., E. Dinerstein, E. D. Wikramanayake, N. D. Burgess, G. V. N. Powell, E. C. Underwood, J. A. D’amico, I. Itoua, H. E. Strand, J. C. Morrison, C. J. Loucks, T. F. Allnutt, T. H. Ricketts, Y. Kura, J. F. Lamoreux, W. W. Wettengel, P. Hedao y K. R. Kassem. 2001. Terrestrial ecoregions of the world: a new map of life on earth. Bioscience 51(11): 933-938. DOI: https://doi.org/10.1641/00063568(2001)051[0933:TEOTWA]2.0.CO;2 
Padilla-Velarde, E., R. Cuevas-Guzmán, G. Ibarra-Manríquez y S. Moreno-Gómez. 2006. Riqueza y biogeografía de la flora arbórea del estado de Colima, México. Revista Mexicana de Biodiversidad 77(2): 271-295. DOI: http:// dx.doi.org/10.22201/ib.20078706e.2006.002.337

Palacio, S., A. Escudero, G. Montserrat-Martí, M. Maestro, R. Milla y M. Albert. 2007. Plants Living on Gypsum: Beyond the Specialist Model. Annals of Botany 99(2): 333-343. DOI: https://doi.org/10.1093/aob/mcl263

Pérez-García, E. A., J. A. Meave, J. L. Villaseñor, J. A. Gallardo-Cruz y E. E. Lebrija-Trejos. 2010. Vegetation heterogeneity and life-strategy diversity in the flora of the heterogeneous landscape of Nizanda, Oaxaca, Mexico. Folia Geobotanica 45(2): 143-161. DOI: https:// doi.org/10.1007/s12224-010-9064-7

PPG I. 2016. A community-derived classification for extant lycophytes and ferns. Journal of Systematics and Evolution 54(6): 563-603. DOI: https://doi.org/10.1111/ jse.12229

Ramírez-Delgadillo, R., J. J. Reynoso-D. y H. J. Arreola-N. 2016. Sapindales. In: Comisión Nacional para el Conocimiento y Uso de la Biodiversidad (ed.). La Biodiversidad en Colima-Estudio de Estado. Comisión Nacional para el Conocimiento y Uso de la Biodiversidad (CONABIO). Cd. Mx., México. Pp. 252-257.

Ramírez-Díaz, C. J. 2016. Estudio florístico del bosque tropical caducifolio en la subcuenca Cuixtla, Jalisco-Zacatecas, México. Tesis de licenciatura. Centro Universitario de Ciencias Biológicas y Agropecuarias, Universidad de Guadalajara. Zapopan, Jalisco, México. 92 pp.

Rivas-Martínez, S. y M. Costa. 1970. Comunidades gipsícolas del centro de España. Anales del Instituto Botánico Cavanilles 27: 193-224.

Rodríguez, A. y L. Ortíz-Catedral. 2003. Colima (Tigridieae: Iridaceae), a new genus from western Mexico and a new species: Colima tuitensis from Jalisco. Acta Botanica Mexicana 65: 51-60. DOI: https://doi.org/10.21829/ abm65.2003.962

Román-Guzmán, T., J. L. Alemán C., J. S. Bracamontes P. y R. Villegas-García. 2016. Cambios en la cobertura vegetal. In: Comisión Nacional para el Conocimiento y Uso de la Biodiversidad (ed.). La Biodiversidad en Colima-Estudio de Estado. Comisión Nacional para el Conocimiento y
Uso de la Biodiversidad (CONABIO). Cd. Mx., México. Pp. 598-605.

Ruiz, J. M., I. López-Cantarero, R. M. Rivero y L. Romero. 2003. Sulphur phytoaccumulation in plant species characteristic of gypsipherous soils. International Journal of Phytoremediation 5(3): 203-210. DOI: https://doi. org/10.1080/713779220

Rzedowski, J. 1955. Notas sobre la flora y la vegetación del estado de San Luis Potosí. II. Estudio de diferencias florísticas y ecológicas condicionadas por ciertos tipos de sustrato geológico. Ciencia (México) 15: 141-158.

Rzedowski, J. 1978. Vegetación de México. Editorial Limusa. México, D.F., México. 432 pp.

Rzedowski, J. 1998. Diversidad y orígenes de la flora fanerogámica de México. In: Ramamoorthy, T. P., R. Bye, A. Lot y J. Fa (comps.). Diversidad biológica de México: orígenes y distribución. Universidad Nacional Autónoma de México. México, D.F., México. Pp. 129-148.

Rzedowski, J. y R. McVaugh. 1966. La Vegetación de Nueva Galicia. Contributions of the University of Michigan Herbarium 9: 1-123.

Santana-Michel, F. J. 2002. Una nueva especie de Aristolochia L., subsección Pentandrae (Aristolochiaceae) del estado de Colima, México. Acta Botanica Mexicana 58: 63-67. DOI: https://doi.org/10.21829/abm58.2002.891

Santana-Michel, F. J., N. Cervantes-Aceves y N. Jiménez-Reyes. 2016. Plantas de importancia apícola. In: Comisión Nacional para el Conocimiento y Uso de la Biodiversidad (ed.). La Biodiversidad de Colima-Estudio de Estado. Comisión Nacional para el Conocimiento y Uso de la Biodiversidad (CONABIO). Cd. Mx., México. Pp. 555-563.

Sáyago-Lorenzana, R., J. Martínez C. y G. Ibarra-M. 2016. Áreas naturales protegidas y prioridades de conservación. In: Comisión Nacional para el Conocimiento y Uso de la Biodiversidad (ed.). La Biodiversidad en Colima-Estudio de Estado. Comisión Nacional para el Conocimiento y Uso de la Biodiversidad (CONABIO). Cd. Mx., México. Pp. 639-646.

SGM. 2010. Carta Geológico-Minera Colima E13-B44, Colima, escala 1: 50,000. Servicio Geológico Mexicano (SGM). Pachuca, México.

SGM. 2019. Carta Geológica de la República Mexicana, escala 1: 1'200,000. Servicio Geológico Mexicano (SGM). Pachuca, México. 
Sousa S., M. 2010. Centros de endemismo: las leguminosas. In: Ceballos, G., L. Martínez, A. García, E. Espinoza, J. Bezaury y R. Dirzo (eds.). Diversidad, amenazas y áreas prioritarias para la conservación de las selvas secas del Pacífico de México. Fondo de Cultura Económica, Comisión Nacional para el Conocimiento y Uso de la Biodiversidad (CONABIO). Cd. Mx., México. Pp. 77-91.

Squeo, F., L. Cavieres, G. Arancio, J. E. Novoa, O. Matthei, C. Marticorena, R. Rodríguez, M. T. K. Arroyo y M. Muñoz. 1998. Biodiversidad de la flora vascular en la región de Antofagasta, Chile. Revista Chilena de Historia Natural 71: 571-591.

Thiers, B. 2020. Index Herbariorum: A Global Directory of Public Herbaria and Associated Staff. New York Botanical Garden's Virtual Herbarium http://sweetgum.nybg.org (consultado julio de 2020).

TNRS. 2020. iPlant Collaborative. Taxonomic Name Resolution Service v. 4.1. http://tnrs.iplantcollaborative.org (consultado diciembre de 2020).

Trejo, I. 2010. Las selvas secas del Pacífico mexicano. In: Ceballos, G., L. Martínez, A. García, E. Espinoza, J. Bezaury y R. Dirzo (eds.). Diversidad, amenazas y áreas prioritarias para la conservación de las selvas secas del Pacífico de México. Fondo de Cultura Económica, Comisión Nacional para el Conocimiento y Uso de la Biodiversidad (CONABIO). Cd. Mx., México. Pp. 41-51.

Trejo, I. y R. Dirzo. 2002. Floristic diversity of Mexican seasonally dry tropical forests. Biodiversity and Conservation 11: 20632084. DOI: https://doi.org/10.1023/A:1020876316013

TROPICOS. 2019. Tropicos.org. Missouri Botanical Garden. Missouri, EUA. http://www.tropicos.org (consultado julio de 2019).

Turner, B. L. y A. M. Powell. 1979. Deserts, gypsum and endemism. In: Goodin, J. R. y D. K. Northington (eds.). Arid Land Plant Resources. International Center for Arid and Semi-Arid Land Studies, Texas Technical University. Lubbock, USA. Pp. 96116.
Ulloa-Ulloa, C., P. Acevedo-Rodríguez, S. G. Beck, M. J. Belgrano, R. Bernal, P. E. Berry, L. Brako, M. Celis, G. Davidse, R. C. Forzza, S. R. Gradstein, O. Hokche, B. León, S. León-Yánez, R. E. Magill, D. A. Neill, M. H. Nee, P. H. Raven, H. Stimmel, M. T. Strong, J. L. Villaseñor, J. L. Zarucchi, F. O. Zuloaga y P. M. Jørgensen. 2017. An integrated assessment of vascular plants species of the Americas. Science 358: 1614-1617. DOI: https://doi.org/10.1126/science.aao0398

Vázquez-García, J. A., M. A. Muñiz-Castro, E. SahagúnGodínez, M. de J. Cházaro-Basañez, E. de Castro-Arce, G. Nieves-Hernández y J. Padilla-Lepe. 2013. Four New Species of Agave (Agavaceae) of the Marmoratae Group. Systematic Botany 38(2): 1-12. DOI: https://doi. org/10.1600/036364413X666642

Villarreal, J. A. y J. A. Encina. 2005. Plantas vasculares endémicas de Coahuila y algunas áreas adyacentes, México. Acta Botanica Mexicana 70: 1-46. DOI: https://doi.org/10.21829/ abm70.2005.986

Villarreal-Quintanilla, J. A., J. A. Bartolomé-Hernández, E. EstradaCastillón, H. Ramírez-Rodríguez y S. J. Martínez-Amador. 2017. El elemento endémico de la flora vascular del Desierto Chihuahuense. Acta Botanica Mexicana 118: 65-96. DOI: http://dx.doi.org/10.21829/abm118.2017.1201

Villaseñor, J. L. 2016. Checklist of the native vascular plants of Mexico. Revista Mexicana de Biodiversidad 87: 559-902. DOI: https://doi.org/10.1016/j.rmb.2016.06.017

Zepeda-Gómez, C. y E. Velázquez-Montes. 1999. El bosque tropical caducifolio de la vertiente sur de la sierra de Nanchititla, estado de México: la composición y la afinidad geográfica de su flora. Acta Botanica Mexicana 46: 29-55. DOI: https:// doi.org/10.21829/abm46.1999.815 
Apéndice 1: Plantas vasculares del bosque tropical caducifolio con suelo yesoso y calcáreo de Colima (La Salada), México. Se enlistan las plantas vasculares con nombres científicos y autores. Se incluye la abreviatura de los nombres de los colectores y sus números de recolecta para cada ejemplar. Los nombres completos de los colectores se presentan en el Cuadro 4. La forma biológica de las plantas vasculares se indica como: $\mathrm{H}=$ hierba; a=arbusto; $\mathrm{A}=a$ rbol; $\mathrm{T}=$ trepadora; $\mathrm{E}=e$ pífita; y $\mathrm{P}=$ parásita. $\left({ }^{*}\right)$ especies endémicas de México; ( $\boldsymbol{\Delta}$ ) taxones conocidos de suelos yesosos y calcáreos; $(\bullet)$ especies con nuevos registros de distribución para el estado de Colima. La mayoría de los ejemplares mencionados se encuentra depositado en el herbario Luz María Villarreal de Puga de la Universidad de Guadalajara (IBUG) y en el herbario de la Universidad de Michigan (MICH) en los Estados Unidos de América. Estos últimos corresponden a colectas históricas de R. McVaugh. Duplicados se han depositado en el Herbario Nacional (MEXU) de la Universidad Nacional Autónoma de México, aunque no de los juegos completos.

\begin{tabular}{ll}
\hline Taxón & Forma biológica \\
\hline
\end{tabular}

\section{PTERIDOFITAS}

\section{Lycopodiopsida}

\section{Selaginellaceae}

Selaginella hoffmannii Hieron.

A Selaginella lepidophylla (Hook. \& Grev.) Spring

$\mathrm{H}$

$\mathrm{H}$

Polypodiopsida

\section{Anemiaceae}

$\Delta$ Anemia adiantifolia (L.) Sw.

* $\Delta$ Anemia colimensis Mickel

$\Delta$ Anemia mexicana var. makrini (Maxon) Mickel

\section{Pteridaceae}

Acrostichum danaefolium Langsd. \& Fisch.

Adiantum tricholepis Fée

*Cheilanthes lozanoi (Maxon) R.Tryon \& A.Tryon var. lozanoi

Cheilanthes lozanoi var. seemannii (Hook.) Mickel \& Beitel

$\Delta$ Cheiloplecton rigidum (Sw.) Fée

Mildella sp.

Notholaena candida (M. Martens \& Galeotti) Hook.

Tectariaceae

$\Delta$ Tectaria heracleifolia (Willd.) Underw.

ANGIOSPERMAS

\section{Magnólidas}

Annonaceae

Annona squamosa $\mathrm{L}$.

\section{Aristolochiaceae}

* $\Delta$ Aristolochia colimensis Santana Mich.

*Aristolochia taliscana Hook. \& Arn.

\section{Hernandiaceae}

Gyrocarpus jatrophifolius Domin

\section{Piperaceae}

*Piper abalienatum Trel.

*Piper jaliscanum S. Watson

\section{Monocotiledóneas}

\section{Amaryllidaceae}

*Hymenocallis proterantha Bauml

Sprekelia formosissima (L.) Herb.
LHL 967, 2290

McV 15526, 15538; LHL 2291

JM 1354; LHL 984

McV 15537, 15539, 26194; JM 1357, 1692

McV 15522, 16056, 18059; JM 1355, 1358, 1691

EL 2755

McV 15521, 16058; McV y K 1547; MH 3440

LHL 640

McV 15536

McV 15523, 18058; McV y K 1071

LHL 966

McV 15539

McV 22970; McV y K 1594

McV y K 1540

FSM 9068

LHL 957

McV y K 1587; MH 3076, 3094A

McV 15564; LHL 882

$\mathrm{MH} 2541$ 
Apéndice 1. Continuación.

\begin{tabular}{|c|c|c|}
\hline Taxón & Forma biológica & Colectas \\
\hline \multicolumn{3}{|l|}{ Asparagaceae } \\
\hline *Agave colimana Gentry & $\mathrm{H}$ & McV 26205; McV y K 1064; AVZ 101 \\
\hline *Agave pablocarrilloi A. Vázquez, Muñiz-Castro \& Padilla-Lepe & $\mathrm{H}$ & JPR 619, EL 915 \\
\hline Echeandia parviflora Baker & $\mathrm{H}$ & RC 1235 \\
\hline${ }^{*}$ Echeandia ramosissima (C.Presl) Cruden & $\mathrm{H}$ & RC 1884, 1986 \\
\hline *Polianthes howardii Verh.-Will. & $\mathrm{H}$ & ARC 3205 \\
\hline \multicolumn{3}{|l|}{ Bromeliaceae } \\
\hline Bromelia pinguin $\mathrm{L}$. & $\mathrm{H}$ & MH 2553 \\
\hline * $\boldsymbol{\Delta}$ Hechtia laevis L.B. Sm. & $\mathrm{H}$ & McV 15528, 16035; LHL 956; MH 3792 \\
\hline *Pitcairnia colimensis L.B. Sm. & $\mathrm{H}$ & McV 15509; AD 377; HI 676; LHL 2286 \\
\hline *Tillandsia achyrostachys E. Morren ex Baker & $\mathrm{E}$ & AVZ 106 \\
\hline Tillandsia balbisiana Schult. f. & $\mathrm{E}$ & McV 24958 \\
\hline Tillandsia caput-medusae E. Morren & $E$ & AVZ 106 \\
\hline *Tillandsia diguetii Mez \& Rol.-Goss. & $E$ & AVZ 107 \\
\hline Tillandsia pseudobaileyi C.S. Gardner & $E$ & AVZ 102 \\
\hline Tillandsia schiedeana Steud. & $\mathrm{E}$ & LHL 782 \\
\hline \multicolumn{3}{|l|}{ Commelinaceae } \\
\hline Commelina erecta $\mathrm{L}$. & $\mathrm{H}$ & LHL 2282 \\
\hline \multicolumn{3}{|l|}{ Cyperaceae } \\
\hline Scleria lithosperma (L.) Sw. & $\mathrm{H}$ & McV 18072 \\
\hline \multicolumn{3}{|l|}{ Dioscoreaceae } \\
\hline${ }^{*}$ Dioscorea mitis C.V. Morton & $\mathrm{T}$ & McV 16039 \\
\hline *Dioscorea oreodoxa B.G. Schub. & $\mathrm{T}$ & McV 16041; MH 4165 \\
\hline *Dioscorea remotiflora Kunth & $\mathrm{T}$ & LHL 1156; MH 3092; MPA 49 \\
\hline Dioscorea subtomentosa Miranda & $\mathrm{T}$ & McV y K 1543; LHL 970 \\
\hline \multicolumn{3}{|l|}{ Iridaceae } \\
\hline *Colima convoluta (Ravenna) Aarón Rodr. \& Ortiz-Catedral & $\mathrm{H}$ & McV 15554; ARC 2960, 4258 \\
\hline \multicolumn{3}{|l|}{ Orchidaceae } \\
\hline *Barkeria palmeri (Rolfe) Schltr. & $\mathrm{H}$ & FH 102/1; MH 3944 \\
\hline *Bletia parkinsonii Hook. & $\mathrm{H}$ & LHL 989 \\
\hline \multicolumn{3}{|l|}{ Poaceae } \\
\hline Aristida adscensionis L. & $\mathrm{H}$ & MH 3935 \\
\hline Bouteloua curtipendula (Michx.) Torr. & $\mathrm{H}$ & McV y K 1545 \\
\hline *Bouteloua elata Reeder \& C. Reeder & $\mathrm{H}$ & McV 18055; JR 2356; LHL 955 \\
\hline Bouteloua media (E. Fourn.) Gould \& Kapadia & $\mathrm{H}$ & McV y K 1545; LHL 2275; MH 3948 \\
\hline A Bouteloua parryi (E. Fourn.) Griffiths & $\mathrm{H}$ & JR 6340; LHL 2276 \\
\hline Eragrostis ciliaris (L.) R.Br. & $\mathrm{H}$ & MH 3937 \\
\hline Eragrostis mexicana (Hornem.) Link & $\mathrm{H}$ & MH 3093 \\
\hline Lasiacis nigra Davidse & $\mathrm{H}$ & LHL 460, 898 \\
\hline Lasiacis ruscifolia (Kunth) Hitchc. var. ruscifolia & $\mathrm{H}$ & McV 18051 \\
\hline Leptochloa virgata (L.) P. Beauv. & $\mathrm{H}$ & $\mathrm{MH} 3083 \mathrm{~A}$ \\
\hline Panicum hirticaule J. Presl & $\mathrm{H}$ & MH 3083 \\
\hline
\end{tabular}


Apéndice 1. Continuación.

\begin{tabular}{|c|c|c|}
\hline Taxón & Forma biológica & Colectas \\
\hline Setaria liebmannii E. Fourn. & $\mathrm{H}$ & JR 4425; LHL 462 \\
\hline Setariopsis auriculata (E. Fourn.) Scribn. & $\mathrm{H}$ & JR 4426 \\
\hline Sorghum bicolor (L.) Moench & $\mathrm{H}$ & McV 18048 \\
\hline Tripsacum dactyloides (L.) L. & $\mathrm{H}$ & LHL 2271 \\
\hline Urochloa maxima (Jacq.) R.D. Webster & $\mathrm{H}$ & MH 3950 \\
\hline \multicolumn{3}{|l|}{ Smilacaceae } \\
\hline Smilax sp. & $\mathrm{T}$ & $\mathrm{MH} 3091 \mathrm{~A}$ \\
\hline \multicolumn{3}{|l|}{ Eudicotiledóneas } \\
\hline \multicolumn{3}{|l|}{ Acanthaceae } \\
\hline *Aphelandra madrensis Lindau & $\mathrm{H}$ & LHL 626; MH 4102 \\
\hline Carlowrightia arizonica A. Gray & $\mathrm{H}$ & LHL 78 \\
\hline Elytraria imbricata (Vahl) Pers. & $\mathrm{H}$ & LHL 1157 \\
\hline Justicia candicans (Nees) L.D. Benson & a & LHL 2288; MH 3452, 3946; MPA 59 \\
\hline *Odontonema auriculatum (Rose) T.F. Daniel & $\mathrm{H}$ & MH 3782 \\
\hline Ruellia inundata Kunth & $\mathrm{H}$ & LHL 81, 638, 655; MH 3947 \\
\hline *•Ruellia longepetiolata (Oerst.) Hemsl. & $\mathrm{H}$ & $\mathrm{MH} 3828$ \\
\hline *Tetramerium mcvaughii T.F. Daniel & a & McV 26191; TD 3247 \\
\hline Tetramerium tenuissimum Rose & a & McV 22959; McV y K 1549 \\
\hline \multicolumn{3}{|l|}{ Amaranthaceae } \\
\hline *Celosia moquini Guill. & $\mathrm{H}$ & MH 3453 \\
\hline Iresine interrupta Benth. & $\mathrm{a}$ & $\mathrm{MH} 4114$ \\
\hline \multicolumn{3}{|l|}{ Anacardiaceae } \\
\hline *Amphipterygium glaucum (Hemls. \& Rose) Standl. & A & MH 3086 \\
\hline *Comocladia engleriana Loes. & A & $\mathrm{MH} 4106$ \\
\hline *Pseudosmodingium perniciosum (Kunth) Engl. & A & LHL 2289; MH 4103 \\
\hline Spondias purpurea L. & A & LHL 789 \\
\hline *•Spondias sp. nov. R. Ramírez D. & A & LHL 784, 965; MH 4109; RRD 4052 \\
\hline \multicolumn{3}{|l|}{ Apiaceae } \\
\hline$*_{\bullet}$ Prionosciadium acuminatum J.M. Coult. \& Rose & $\mathrm{H}$ & McV 15566, 16057 \\
\hline \multicolumn{3}{|l|}{ Apocynaceae } \\
\hline Cascabela ovata (Cav.) Lippold & A & McV 1532, 1533, 15572; LHL 897; MH 3087A \\
\hline${ }^{*}$ Funastrum pannosum Schltr. & $\mathrm{T}$ & LHL 649, 777; MH 3078 \\
\hline Haplophyton cimicidum A. DC. & $\mathrm{H}$ & LHL 451, 628; MH 2542 \\
\hline *Mandevilla exilicaulis (Sessé \& Moc.) J.K. Williams & $\mathrm{T}$ & MH 3936 \\
\hline *Marsdenia coulteri Hemsl. & $\mathrm{T}$ & LHL 646, 648; MH 2554 \\
\hline *•Marsdenia sp. nov. V. Juárez & $\mathrm{T}$ & $\mathrm{MH} 3422 \mathrm{~A}$ \\
\hline Plumeria rubra L. & A & LHL 795A, 891 \\
\hline Tabernaemontana donnell-smithii Rose & A & MH 3094, 3450, 3825 \\
\hline \multicolumn{3}{|l|}{ Asteraceae } \\
\hline *Adenophyllum aurantium (L.) Strother & $\mathrm{H}$ & McV y K 1059 \\
\hline - Bidens reptans var. urbanii (Greenm.) O.E. Schulz & $\mathrm{a}$ & McV y K 1485; MH 3469 \\
\hline
\end{tabular}


Apéndice 1. Continuación.

\begin{tabular}{|c|c|c|}
\hline Taxón & Forma biológica & Colectas \\
\hline Brickellia coulteri A. Gray & a & LHL 636; MH 3467A \\
\hline Brickellia diffusa (Vahl) A. Gray & $\mathrm{H}$ & LHL 968; MH 3467 \\
\hline *Chromolaena haenkeana (DC.) R.M. King \& H. Rob. & $\mathrm{H}$ & McV y K 1593 \\
\hline Chromolaena odorata (L.) R.M. King \& H. Rob. & $\mathrm{H}$ & McV y K 1047; MH 3424 \\
\hline *Cymophora accedens (S.F. Blake) B.L. Turner \& A.M. Powell & $\mathrm{H}$ & MH 3079 \\
\hline * $\mathbf{\Delta}$ Flaveria robusta Rose & $\mathrm{H}$ & McV 22943; McV y K 1044; LHL 788; MH 3785, 4169 \\
\hline $\boldsymbol{\Delta}$ Lagascea mollis Cav. & $\mathrm{H}$ & LHL 2280; MH 3458 \\
\hline * $\mathbf{\Delta}$ Lagascea palmeri (B.L. Rob.) B.L. Rob. & a & McV y K 104808; LHL 2270; MH 3430 \\
\hline *Lasianthaea ceanothifolia (Willd.) K.M. Becker & $\mathrm{a}$ & LHL 2277 \\
\hline * $\Delta$ Lepidaploa koelzii (McVaugh) H. Rob. & a & McV y K 1576 \\
\hline * $\mathbf{\Delta}$ Melampodium nutans Stuessy & $\mathrm{H}$ & McV 18070; McV y K 1054; LHL 983; MH 3077, 3945 \\
\hline *Montanoa laskowskii McVaugh & a & McV 18053; LHL 2281; MH 3420 \\
\hline *AOtopappus koelzii McVaugh & a & McV y K 1066 \\
\hline *Pittocaulon filare (McVaugh) H. Rob. \& Brettell & A & McV 26200, 26296; McV y K 1550; LHL 990; MH 3796 \\
\hline Porophyllum punctatum (Mill.) S.F. Blake & a & McV y K 1577; LHL 1159; MH 3081 \\
\hline Porophyllum ruderale var. macrocephalum (DC.) Cronquist & $\mathrm{H}$ & $\mathrm{MH} 3425$ \\
\hline *Sinclairia liebmannii (Klatt) Sch. Bip. ex Rydb. & $\mathrm{a}$ & MH 3427; 3446 \\
\hline Tithonia rotundifolia (Mill.) S.F. Blake & $\mathrm{H}$ & LHL 2279 \\
\hline *Trixis mexicana Lex. var. mexicana & a & FSM 138; MH 3423, 3457, 4096 \\
\hline * $\boldsymbol{\Delta}$ Verbesina mickelii McVaugh & a & McV 16054, 18050, 24962; LHL 976; MH 3080, 3421 \\
\hline *Verbesina platyptera Sch. Bip. ex Klatt & $\mathrm{H}$ & McV y K 1548; MH 3434, 3827, 4097 \\
\hline A Viguiera dentata (Cav.) Spreng. & $\mathrm{H}$ & McV 22965, 24964; McV y K 1079; MH 4170 \\
\hline *Zinnia zinnioides (Kunth) Olorode \& A.M. Torres & $\mathrm{H}$ & LHL 2278 \\
\hline \multicolumn{3}{|l|}{ Begoniaceae } \\
\hline *Begonia monophylla Pav. ex A. DC. & $\mathrm{H}$ & McV 15568, 18067; McV y K 1068, 1572; LHL 971, 2292 \\
\hline \multicolumn{3}{|l|}{ Bignoniaceae } \\
\hline Crescentia alata Kunth & A & $\mathrm{MH} 3080 \mathrm{~A}$ \\
\hline Handroanthus chrysanthus (Jacq.) S.O. Grose & A & $\mathrm{MH} 3470 \mathrm{~A}$ \\
\hline Tabebuia rosea (Bertol.) DC. & A & $\mathrm{MH} 3081 \mathrm{~A}$ \\
\hline \multicolumn{3}{|l|}{ Bixaceae } \\
\hline Cochlospermum vitifolium (Willd.) Spreng. & A & LHL 653, 977 \\
\hline \multicolumn{3}{|l|}{ Burseraceae } \\
\hline *Bursera arborea (Rose) L. Riley & A & McV 15558 \\
\hline *Bursera citronella McVaugh \& Rzed. & A & McV 15557, 16045; MH 3087 \\
\hline *Bursera denticulata McVaugh \& Rzed. & A & McV 15525, 16046 \\
\hline Bursera excelsa (Kunth) Engl. & A & LHL 2274; MH 3082A \\
\hline${ }^{*}$ Bursera palmeri S. Watson & A & LHL 884 \\
\hline *Bursera penicillata (DC.) Engl. & A & $\mathrm{MH} 3471 \mathrm{~A}$ \\
\hline *Bursera sarcopoda Paul G. Wilson & A & McV y K 1445, 1452, 1564; MH 3829, 3943, 3088A \\
\hline Bursera schlechtendalii Engl. & A & McV 15559 \\
\hline \multicolumn{3}{|l|}{ Cactaceae } \\
\hline *Cephalocereus nudus E.Y. Dawson & A & Observado \\
\hline
\end{tabular}


Apéndice 1. Continuación.

\begin{tabular}{|c|c|c|}
\hline Taxón & Forma biológica & Colectas \\
\hline *Opuntia karwinskiana Salm-Dyck & $A$ & MH 3471 \\
\hline \multicolumn{3}{|l|}{ Capparaceae } \\
\hline Cynophalla verrucosa (Jacq.) J. Presl & A & McV 15565, 22960; LHL 465, 1161; MH 3786 \\
\hline *Forchhammeria pallida Liebm. & $A$ & LHL 650; MH 3830, 3083A \\
\hline Quadrella incana (Kunth) Iltis \& Cornejo & A & McV 22946; LHL 1166; MH 3795, 3819, 3820 \\
\hline \multicolumn{3}{|l|}{ Caprifoliaceae } \\
\hline *Valeriana palmeri A. Gray & $\mathrm{H}$ & McV 18057 \\
\hline \multicolumn{3}{|l|}{ Celastraceae } \\
\hline Crossopetalum parviflorum (Hemsl.) Lundell & a & LHL 791, 986; MH 3466, 4164 \\
\hline Pristimera celastroides (Kunth) A.C. Sm. & $\mathrm{T}$ & $\mathrm{MH} 4104 \mathrm{~A}$ \\
\hline *Wimmeria lanceolata Rose & A & $\mathrm{MH} 3086 \mathrm{~A}$ \\
\hline \multicolumn{3}{|l|}{ Cleomaceae } \\
\hline Physostemon humilis (Rose) Iltis & $\mathrm{H}$ & LHL 625 \\
\hline \multicolumn{3}{|l|}{ Combretaceae } \\
\hline *Combretum igneiflorum Rendón \& R. Delgad. & $\mathrm{T}$ & LHL 972; MH 3776 \\
\hline Combretum fruticosum (Loefl.) Stuntz & $\mathrm{T}$ & MH 3468 \\
\hline \multicolumn{3}{|l|}{ Convolvulaceae } \\
\hline *Calycobolus nutans (Moc. \& Sessé ex Choisy) D.F. Austin & $\mathrm{T}$ & McV 24965 \\
\hline - Cuscuta macvaughii Yunck. & $\mathrm{P}$ & LHL 672; MH 2550, 3941 \\
\hline Ipomoea arborescens (Humb. \& Bonpl. ex Willd.) G. Don. & $A$ & McV y K 1582 \\
\hline *Ipomoea bracteata Cav. & $\mathrm{T}$ & LHL 790 \\
\hline Ipomoea hederifolia $\mathrm{L}$. & $\mathrm{T}$ & McV 18080 \\
\hline Ipomoea nil (L.) Roth & $\mathrm{T}$ & McV 18047 \\
\hline Ipomoea praecana House & $\mathrm{T}$ & McV 22972; McV y K 1567 \\
\hline Ipomoea ternifolia Cav. & $\mathrm{T}$ & LHL 80 \\
\hline Ipomoea wolcottiana Rose & A & McV 22968; EC 6038; MH 4107 \\
\hline *Jacquemontia nelsonii House & $\mathrm{T}$ & LHL 621, 2269; MH 3448 \\
\hline Jacquemontia pentanthos (Jacq.) G. Don & $\mathrm{T}$ & LHL 620; 2268 \\
\hline -Jacquemontia polyantha (Schltdl. \& Cham.) Hallier f. & $\mathrm{T}$ & McV \& K 1075 \\
\hline *Merremia platyphylla (Fernald) O’Donell & $\mathrm{T}$ & LHL 659; MH 3942 \\
\hline Merremia quinquefolia (L.) Hallier f. & $\mathrm{T}$ & McV y K 1596 \\
\hline Merremia umbellata (L.) Hallier $\mathrm{f}$. & $\mathrm{T}$ & LHL 79 \\
\hline Operculina pteripes (G. Don) O’Donell & $\mathrm{T}$ & McV 16060 \\
\hline \multicolumn{3}{|l|}{ Cordiaceae } \\
\hline Cordia alliodora (Ruiz \& Pav.) Oken & $A$ & LHL 962; MH 3422 \\
\hline${ }^{*}$ Cordia seleriana Fernald & $A$ & LHL 77; MH 3775, 3787, 4160 \\
\hline \multicolumn{3}{|l|}{ Cucurbitaceae } \\
\hline Cyclanthera gracillima Cogn. & $\mathrm{T}$ & McV 24961; JD 3537; MPA 40 \\
\hline Melothria pendula L. & $\mathrm{T}$ & MPA 44 \\
\hline \multicolumn{3}{|l|}{ Euphorbiaceae } \\
\hline A Acalypha alopecuroidea Jacq. & $\mathrm{H}$ & LHL 447 \\
\hline * $\boldsymbol{\Delta}$ Acalypha umbrosa Brandegee & $\mathrm{H}$ & LHL 889 \\
\hline
\end{tabular}


Apéndice 1. Continuación.

\begin{tabular}{|c|c|c|}
\hline Taxón & Forma biológica & Colectas \\
\hline *Acalypha vagans Cav. & a & McV 15546 \\
\hline *Adelia oaxacana (Müll. Arg.) Hemsl. & $\mathrm{T}$ & MH 2517 \\
\hline * $\mathbf{\Delta}$ Bernardia spongiosa McVaugh & A & McV 22971 \\
\hline$* \boldsymbol{\Delta}$ Cnidoscolus spinosus Lundell & A & McV 18049, LHL 896 \\
\hline A Croton fragilis Kunth & a & McV 15524, LHL 878 \\
\hline Croton morifolius Willd. & a & McV 15507 \\
\hline Croton pseudoniveus Lundell & A & LHL 647; MH 3438 \\
\hline$\Delta$ Croton reflexifolius Kunth & A & MH 2527, 3818 \\
\hline * $\boldsymbol{\Delta}$ Croton suberosus Kunth & a & LHL 980; MH 3088 \\
\hline * $\boldsymbol{\Delta}$ Dalembertia populifolia Baill. & $\mathrm{T}$ & McV 18081; McV y K 1045; MH 3084A \\
\hline Ditaxis guatemalensis (Müll. Arg.) Pax \& K. Hoffm. & a & McV 18063 \\
\hline * $\mathbf{\Delta}$ Euphorbia calcarata (Schltdl.) V.W. Steinm. & A & McV 22954; LHL 982; MH 3085A \\
\hline Euphorbia colletioides Benth. & $\mathrm{H}$ & LHL 1154 \\
\hline Euphorbia cyathophora Murray & $\mathrm{H}$ & McV y K 1078 \\
\hline Euphorbia delicatula Boiss. & $\mathrm{H}$ & McV y K 1070 \\
\hline Euphorbia graminea Jacq. & $\mathrm{H}$ & LHL 634, 959, 987; MH 3426, 3442 \\
\hline Euphorbia heterophylla L. & $\mathrm{H}$ & LHL 985 \\
\hline Euphorbia hirta L. & $\mathrm{H}$ & LHL 450, 883 \\
\hline Euphorbia humayensis Brandegee & $\mathrm{H}$ & McV y K 1055 \\
\hline Euphorbia hypericifolia L. & $\mathrm{H}$ & McV 16052; LHL 452 \\
\hline Euphorbia nutans Lag. & $\mathrm{H}$ & LHL 892 \\
\hline *Euphorbia oaxacana B.L. Rob. \& Greenm. & $\mathrm{T}$ & McV y K 1580; MH 3789; PCR 3041 \\
\hline A Euphorbia schlechtendalii Boiss. var. websteri McVaugh & $\mathrm{T}$ & McV 26199; McV y K 1591; CGM 10; MH 2516, 2545 \\
\hline *Euphorbia strigosa Hook. \& Arn. & $\mathrm{H}$ & McV y K 1058, 1068 \\
\hline *Jatropha platyphylla Müll. Arg. & A & MH 3079A \\
\hline Manihot angustiloba (Torr.) Müll. Arg. & a & McV 15531 \\
\hline *A Manihot chlorosticta Standl. \& Goldman & $\mathrm{H}$ & McV y K 1533; MH 3432 \\
\hline *Manihot michaelis McVaugh & A & MH 3078A \\
\hline Manihot rhomboidea Müll. Arg. & a & McV 15571 \\
\hline \multicolumn{3}{|l|}{ Fabaceae } \\
\hline Acaciella angustissima (Mill.) Britton \& Rose & A & McV 16042 \\
\hline Acaciella painteri var. houghii (Britton \& Rose) L. Rico & A & LHL 463 \\
\hline Acaciella tequilana S. Watson var. pubifoliolata L.Rico & a & LHL 671, 2284 \\
\hline Aeschynomene amorphoides (S. Watson) Rose ex B.L. Rob. & a & LHL 642; MH 3437 \\
\hline Aeschynomene histrix Poir. & a & LHL 2285 \\
\hline Bauhinia divaricata $\mathrm{L}$. & A & McV 22964; McV y K 1598; MH 3443 \\
\hline Bauhinia gypsicola McVaugh & A & McV y K 1539 \\
\hline Bauhinia subrotundifolia Cav. & A & McV 26187; LHL 954A; MH 3781 \\
\hline Caesalpinia cacalaco Bonpl. & A & McV y K 1554, 1555; MH 4118, 4173 \\
\hline Calliandra calothyrsus Meisn. & A & McV 16062 \\
\hline *Calliandra hirsuta (G. Don) Benth. & A & McV 15550; McV y K 1043; MH 3085, 3449A \\
\hline
\end{tabular}


Apéndice 1. Continuación.

\begin{tabular}{|c|c|c|}
\hline Taxón & Forma biológica & Colectas \\
\hline $\begin{array}{l}\text { Calliandra tergemina var. emarginata (Humb. \& Bonpl. ex } \\
\text { Willd.) Barneby }\end{array}$ & a & $\mathrm{MH} 2525,3421 \mathrm{~A}$ \\
\hline Canavalia brasiliensis Mart. ex Benth. & $\mathrm{T}$ & LHL 991; MPA 57 \\
\hline *Cassia hintonii Sandwith & A & LHL 783, 894 \\
\hline Centrosema virginianum (L.) Benth. & $\mathrm{T}$ & LHL 624 \\
\hline${ }^{*}$ Coulteria platyloba (S. Watson) N. Zamora & A & MH 2537 \\
\hline A Coursetia caribaea (Jacq.) Lavin & A & McV y K 1575 \\
\hline Coursetia glandulosa A. Gray & A & MH 4108 \\
\hline *Coursetia mollis B.L. Rob. \& Greenm. & A & MH 2524; MPA 46 \\
\hline *•Coursetia oaxacensis M.Sousa \& Rudd & a & LHL 643A; MH 3447 \\
\hline Desmanthus bicornutus S. Watson & $\mathrm{H}$ & LHL 641 \\
\hline Desmodium glabrum (Mill.) DC. & $\mathrm{H}$ & MH 3441 \\
\hline * $\mathbf{\Delta}$ Diphysa occidentalis Rose & A & McV y K 1588; LHL 629; MH 3824 \\
\hline $\begin{array}{l}\text { *Erythrina lanata subsp. occidentalis (Standl.) Krukoff \& } \\
\text { Barneby }\end{array}$ & A & LHL 895 \\
\hline Galactia acapulcensis Rose & $\mathrm{T}$ & McV y K 1566 \\
\hline * $\Delta$ Galactia viridiflora (Rose) Standl. & $\mathrm{T}$ & McV y K 1579 \\
\hline Gliricidia sepium (Jacq.) Kunth ex Walp. & A & McV 22944; McV y K 1050; LHL 781; MH 2536, 3470, 4163 \\
\hline Haematoxylum brasiletto H. Karst. & A & McV 22966; LHL 76; MH 3472A \\
\hline * $\boldsymbol{\Delta}$ Indigofera palmeri S. Watson & A & McV 16055; LHL 464 \\
\hline *Leucaena lanceolata S. Watson & A & LHL 645 \\
\hline * $\mathbf{\Delta}$ Lonchocarpus eriocarinalis Micheli & A & JASM 2345; MH 3089A \\
\hline Lonchocarpus lanceolatus Benth. & A & McV 15555 \\
\hline *Lonchocarpus mutans M. Sousa & A & JASM 2346 \\
\hline *Lysiloma tergeminum Benth. & A & McV 16036 \\
\hline Macroptilium gracile (Benth.) Urb. & $\mathrm{T}$ & MH 3436 \\
\hline *Marina neglecta (B.L. Rob.) Barneby & $\mathrm{H}$ & McV 26189; McV y K 1073; LHL 1167, 2283; MH 3784 \\
\hline *Marina unifoliata (B.L. Rob. \& Greenm.) Barneby & H & MH 3429 \\
\hline *Mimosa acantholoba (Humb. \& Bonpl. ex Willd.) Poir & A & McV y K 1560 \\
\hline *Mimosa rosei B.L. Rob. & A & McV 15544; McV y K 1586; LHL 459, 631 \\
\hline *Mimosa sicyocarpa B.L. Rob. & a & McV 18054, 18068 \\
\hline *Nissolia leiogyne Sandwith & $\mathrm{T}$ & McV 18052; MH 3084; W y B 16090 \\
\hline $\begin{array}{l}\text { Pachyrhizus erosus (L.) Urb. var. palmatilobus (DC.) R.T. } \\
\text { Clausen }\end{array}$ & $\mathrm{T}$ & McV 16059; SK 2785 \\
\hline *Phaseolus macvaughii Delgado & $\mathrm{T}$ & MH 3435 \\
\hline A Piscidia grandifolia var. glabrescens Sandwith & A & McV y K 1571; FMG 91113 \\
\hline *Platymiscium lasiocarpum Sandwith & A & LHL 1153; MH 3420A \\
\hline Poeppigia procera C. Presl & A & LHL 880 \\
\hline Poincianella eriostachys (Benth.) Britton \& Rose & A & McV 22974; MH 4161 \\
\hline *Pterocarpus orbiculatus DC. & A & MH 2555 \\
\hline Ramirezella strobilophora (B.L. Rob. ex Pringle) Rose & $\mathrm{T}$ & MH 2530, 4113, 4174 \\
\hline Rhynchosia minima (L.) DC. & $\mathrm{H}$ & $\mathrm{MH} 2535,3428$ \\
\hline
\end{tabular}


Apéndice 1. Continuación.

\begin{tabular}{|c|c|c|}
\hline Taxón & Forma biológica & Colectas \\
\hline *Senegalia macilenta (Rose) Britton \& Rose & A & McV 22963; LHL 1164; MH 3778, 4111 \\
\hline Senna atomaria (L.) H.S. Irwin \& Barneby & A & LHL 978; MH 4162 \\
\hline Senna pallida (Vahl) H.S. Irwin \& Barney & A & McV y K 1559; MH 2522, 4104 \\
\hline Senna racemosa var. coalcomanica H.S. Irwin \& Barneby & A & McV y K 1578; LHL 1170 \\
\hline Senna wislizeni var. pringlei (Rose) H.S. Irwin \& Barneby & A & McV 16034; McV y K 1585; LHL 449, 632 \\
\hline Vachellia campechiana (Mill.) Seigler \& Ebinger & A & MH 3461 \\
\hline Vachellia hindsii (Benth.) Seigler \& Ebinger & $A$ & MH 3780 \\
\hline $\begin{array}{l}\text { Zapoteca formosa (Kunth) H.M. Hern. subsp. rosei (Wiggins) } \\
\text { H.M. Hern. }\end{array}$ & A & LHL 639 \\
\hline \multicolumn{3}{|l|}{ Gentianaceae } \\
\hline Centaurium quitense (Kunth) B.L. Rob. & $\mathrm{H}$ & McV y K 1562 \\
\hline Eustoma exaltatum (L.) Salisb. ex G. Don & $\mathrm{H}$ & LHL 1165 \\
\hline \multicolumn{3}{|l|}{ Heliotropiaceae } \\
\hline Heliotropium angiospermum Murray & $\mathrm{H}$ & MH 2539, 3465 \\
\hline Heliotropium fallax I.M. Johnst. & $\mathrm{H}$ & McV 18072 \\
\hline Heliotropium fruticosum $\mathrm{L}$. & $\mathrm{H}$ & LHL 886 \\
\hline Tournefortia mutabilis Vent. & a & $\mathrm{MH} 2518,3454,3464,3946 \mathrm{~A}$ \\
\hline Tournefortia volubilis $\mathrm{L}$. & a & LHL 881 \\
\hline \multicolumn{3}{|l|}{ Lamiaceae } \\
\hline Hyptis suaveolens (L.) Poit. & $\mathrm{H}$ & $\mathrm{MH} 3444$ \\
\hline Hyptis subtilis Epling & a & McV y K 1049; MH 3439, 3460 \\
\hline *Salvia languidula Epling & $\mathrm{H}$ & LHL 974 \\
\hline \multicolumn{3}{|l|}{ Lentibulariaceae } \\
\hline *A Pinguicula colimensis McVaugh \& Mickel & $\mathrm{H}$ & McV 15534, 18065; McV y K 1072; LHL 2273; SZR 9132 \\
\hline \multicolumn{3}{|l|}{ Loranthaceae } \\
\hline Struthanthus interruptus (Kunth) G. Don & $\mathrm{P}$ & MH 3777 \\
\hline \multicolumn{3}{|l|}{ Lythraceae } \\
\hline Cuphea leptopoda Hemsl. & $\mathrm{H}$ & McV 16051 \\
\hline \multicolumn{3}{|l|}{ Malpighiaceae } \\
\hline *Bunchosia palmeri S. Watson & A & LHL 644, 658; MH 3076 \\
\hline Callaeum macropterum (Moc. \& Sessé ex DC.) D.M. Johnson & $\mathrm{T}$ & McV 22949; LHL 1155 \\
\hline${ }^{*}$ Echinopterys eglandulosa (A. Juss.) Small & $\mathrm{T}$ & FSM 140; MH 2540 \\
\hline *Gaudichaudia mcvaughii W.R. Anderson & $\mathrm{T}$ & McV 16043; LHL 456; MH 3433; WA 12699, 12703 \\
\hline *Malpighia novogaliciana W.R. Anderson & a & McV 15549 \\
\hline *Malpighia rzedowskii W.R. Anderson & a & McV 16037; JR y McV 1409 \\
\hline *Tetrapterys mexicana Hook. \& Arn. & $\mathrm{T}$ & McV 23235, 22947; WA 5997 \\
\hline \multicolumn{3}{|l|}{ Malvaceae } \\
\hline Abutilon abutiloides (Jacq.) Garcke ex Hochr. & $\mathrm{H}$ & SK 89178; WA 5997 \\
\hline * $\Delta$ Abutilon bastardioides Baker f. ex Rose & a & McV y K 1042; FSM 1200; MH 2514; AS 11935; SK 89177 \\
\hline Ayenia filiformis S. Watson & a & LHL 630; GW 16130 \\
\hline Ayenia micrantha Standl. & a & McV 16040 \\
\hline Ayenia wrightii B.L. Rob. & a & McV y K 1589; MH 3459 \\
\hline
\end{tabular}


Apéndice 1. Continuación.

\begin{tabular}{|c|c|c|}
\hline Taxón & Forma biológica & Colectas \\
\hline${ }^{*} \boldsymbol{\Delta}$ Bastardiastrum batesii Fryxell \& S.D. Koch & a & FSM 894; MH 2549; SK 89173 \\
\hline *Bastardiastrum hirsutiflorum (C. Presl) D.M. Bates & a & McV y K 1052, 1581, 22945; MH 2548; W y B 16092 \\
\hline *Bastardiastrum incanum (Brandegee) D.M. Bates & a & LHL 981, 1162 \\
\hline $\begin{array}{l}\text { *Bastardiastrum tricarpellatum (B.L. Rob. \& Greenm.) D.M. } \\
\text { Bates }\end{array}$ & a & FSM 136 \\
\hline Gaya minutiflora Rose & $\mathrm{H}$ & LHL 793 \\
\hline *Gossypium aridum (Rose \& Standl.) Skovst. & A & $\begin{array}{l}\text { McV 15543, 22952; McV y K 1041; FSM 135; LHL 82, 654, } \\
\text { 1168; AS } 11751\end{array}$ \\
\hline *Gossypium lanceolatum Tod. & a & RA 1688 \\
\hline Heliocarpus occidentalis Rose & A & McV 18069; LHL 637 \\
\hline Herissantia crispa (L.) Brizicky & a & MH 2547 \\
\hline${ }^{*}$ Hibiscus citrinus Fryxell & a & MH 2544 \\
\hline $\mathbf{\Delta}$ Malvastrum americanum (L.) Torr. & $\mathrm{H}$ & SK 89170 \\
\hline Pseudobombax ellipticum (Kunth) Dugand & A & LHL 775 \\
\hline Sida abutilifolia Mill. & $\mathrm{H}$ & AS 11755 \\
\hline Sida ciliaris L. & $\mathrm{H}$ & AS 11756 \\
\hline * $\boldsymbol{\Delta}$ Waltheria bicolor J.G. Saunders & a & McV y K 1077; LHL 623, 637, 657; GW 16120; MH 3939 \\
\hline \multicolumn{3}{|l|}{ Meliaceae } \\
\hline Swietenia humilis Zucc. & A & LHL 1172 \\
\hline Trichilia americana (Sessé \& Moc.) T.D. Penn. & A & LHL 635 \\
\hline \multicolumn{3}{|l|}{ Menispermaceae } \\
\hline *Hyperbaena ilicifolia Standl. & $\mathrm{T}$ & $\mathrm{MH} 3469 \mathrm{~A}$ \\
\hline \multicolumn{3}{|l|}{ Moraceae } \\
\hline Ficus cotinifolia Kunth & A & McV 15527; LHL 786, 988 \\
\hline Ficus obtusifolia Kunth & A & LHL 991A \\
\hline *Ficus pringlei S. Watson & A & LHL 785, 991B; MH 4167, 4172 \\
\hline \multicolumn{3}{|l|}{ Muntingiaceae } \\
\hline Muntingia calabura L. & A & LHL 1169 \\
\hline \multicolumn{3}{|l|}{ Nyctaginaceae } \\
\hline Boerhavia sp. & $\mathrm{H}$ & McV 16049 \\
\hline Commicarpus scandens (L.) Standl. & $\mathrm{H}$ & LHL 958; MH 2543 \\
\hline - Grajalesia fasciculata (Standl.) Miranda & $A$ & McV 22948 \\
\hline Mirabilis sp. & $\mathrm{H}$ & McV 16048 \\
\hline Pisonia aculeata $\mathrm{L}$. & A & LHL 964, 1171; MH 2523 \\
\hline \multicolumn{3}{|l|}{ Opiliaceae } \\
\hline Agonandra racemosa (DC.) Standl. & A & McV 22950 \\
\hline \multicolumn{3}{|l|}{ Orobanchaceae } \\
\hline *Lamourouxia colimae W.R. Ernst \& Baad & $\mathrm{H}$ & McV 18077 \\
\hline \multicolumn{3}{|l|}{ Oxalidaceae } \\
\hline Oxalis frutescens $\mathrm{L}$. & $\mathrm{H}$ & LHL 454 \\
\hline Oxalis latifolia Kunth & $\mathrm{H}$ & LHL 885 \\
\hline
\end{tabular}


Apéndice 1. Continuación.

Taxón $\quad$ Forma biológica Colectas

\section{Passifloraceae}

Passiflora mexicana Juss.

Turnera diffusa Willd.

Phyllanthaceae

* $\mathbf{\Delta}$ Phyllanthus gypsicola McVaugh

*Phyllanthus standleyi McVaugh

\section{Picramniaceae}

Alvaradoa amorphoides Liebm.

\section{Plantaginaceae}

*Russelia retrorsa Greene

Russelia sarmentosa Jacq.

${ }^{*}$ Russelia tenuis Lundell

Polemoniaceae

Loeselia glandulosa (Cav.) G. Don

Loeselia pumila (M. Martens \& Galeotti) Walp.

Polygalaceae

*Hebecarpa rivinifolia (Kunth) J.R. Abbott \& J.F.B. Pastore

\section{Polygonaceae}

Antigonon flavescens S. Watson

*Coccoloba jurgenseni Lindau

Primulaceae

Bonellia nervosa (C.Presl) B. Ståhl \& Källersjö

\section{Rhamnaceae}

Colubrina elliptica (Sw.) Brizicky \& W.L. Stern.

Colubrina triflora Brongn. ex G. Don

Gouania lupuloides (L.) Urb.

* Sarcomphalus amole (Sessé \& Moc.) Hauenschild

* Sarcomphalus mexicanus (Rose) Hauenschild

\section{Rubiaceae}

*Bouvardia loeseneriana Standl.

Exostema caribaeum (Jacq.) Schult.

*•Guettarda filipes Standl.

Hintonia sp.

Randia aculeata $\mathrm{L}$.

\section{Rutaceae}

Esenbeckia sp.

*Zanthoxylum arborescens Rose

Zanthoxylum mollisimum (Engl.) P. Wilson

Sapindaceae

Paullinia clavigera Schltdl.
$\mathrm{T}$

$\mathrm{H}$

LHL 455

McV 18069; AFC 1991

McV 15542; McV y K 1573

McV 16038

A

McV 22958; McV y K 1062; LHL 960

H $\quad$ LHL 961

$\mathrm{H}$

MH 3472; MPA 58

MH 3790

H LHL 975

H LHL 627

a

LHL 458; MH 3456

T

MPA 43

A

LHL 969

McV 22969; McV y K 1552; LHL 443, 652, 778, 887; MH 3431

A

McV y K 1595

A

LHL 651, 963; MH 3938

$\mathrm{T}$

MH 3462

A

MH 3794

A

McV y K 1574; LHL 794, 2008; MH 3092A

a

McV 18060; McV y K 1046; MPA 55; PZT 55

LHL 622

A

McV 15561

A

McV 15535, 15553

a

LHL 622; MH 2531

A

LHL 877

A McV 18062

A

McV 15562, 18066; MH 3090, 3821 
Apéndice 1. Continuación.

\begin{tabular}{|c|c|c|}
\hline Taxón & Forma biológica & Colectas \\
\hline Paullinia fuscescens Kunth & $\mathrm{T}$ & McV 22951; LHL 1163; MH 2526 \\
\hline Serjania racemosa Schumach. & $\mathrm{T}$ & MH 3783, 3940 \\
\hline *Serjania schiedeana Schltdl. & $\mathrm{T}$ & MH 3445, 3463 \\
\hline *Thouinia serrata Radlk. & A & MH 3822 \\
\hline *Thouinia villosa DC. & A & MH 2515 \\
\hline \multicolumn{3}{|l|}{ Sapotaceae } \\
\hline Sideroxylon capiri subsp. tempisque (Pittier) T.D. Penn. & A & LHL 1158 \\
\hline \multicolumn{3}{|l|}{ Solanaceae } \\
\hline Datura discolor Bernh. & $\mathrm{H}$ & MH 3089 \\
\hline Solanum hazenii Britton & a & LHL 461, 879 \\
\hline *Solanum houstonii Martyn & a & MH 3082 \\
\hline \multicolumn{3}{|l|}{ Talinaceae } \\
\hline Talinum sp. & $\mathrm{H}$ & LHL 780 \\
\hline \multicolumn{3}{|l|}{ Urticaceae } \\
\hline Myriocarpa longipes Liebm. & a & LHL 457; MH 4118A \\
\hline $\begin{array}{l}\text { Pouzolzia occidentalis var. palmeri (S.Watson) Friis \& Wilmot- } \\
\text { Dear }\end{array}$ & A & McV 15563 \\
\hline \multicolumn{3}{|l|}{ Verbenaceae } \\
\hline Citharexylum standleyi Moldenke & A & McV 15551 \\
\hline Lantana achyranthifolia Desf. & a & LHL 444, 633 \\
\hline *Lantana langlassei Moldenke & a & McV 18061; LHL 453 \\
\hline Lippia umbellata Cav. & A & McV y K 1069; LHL 446 \\
\hline \multicolumn{3}{|l|}{ Violaceae } \\
\hline *Hybanthus serrulatus Standl. & $\mathrm{H}$ & MH 2520 \\
\hline Pombalia attenuata (Humb. \& Bonpl. ex Willd.) Paula-Souza & $\mathrm{H}$ & McV 16050 \\
\hline \multicolumn{3}{|l|}{ Vitaceae } \\
\hline Ampelopsis denudata Planch. & $\mathrm{T}$ & MH 3091 \\
\hline Cissus verticillata (L.) Nicolson \& C.E. Jarvis & $\mathrm{T}$ & LHL 75 \\
\hline -Vitis cinerea (Engelm.) Millardet & $\mathrm{T}$ & MH 3077A \\
\hline Vitis tiliifolia Humb. \& Bonpl. ex Schult. & $\mathrm{T}$ & LHL 776 \\
\hline \multicolumn{3}{|l|}{ Zygophyllaceae } \\
\hline Kallstroemia grandiflora Torr. ex A. Gray & $\mathrm{H}$ & LHL 448 \\
\hline Kallstroemia pubescens (G. Don) Dandy & $\mathrm{H}$ & McV 18078 \\
\hline *Kallstroemia rosei Rydb. & $\mathrm{H}$ & CGM 9, 2; LHL 787 \\
\hline
\end{tabular}


Apéndice 2: Plantas vasculares del bosque tropical caducifolio con sustrato yesoso en el municipio Coquimatlán, Colima, México. Los registros de este apéndice fueron obtenidos y depurados a partir de la consulta de bases de datos electrónicas como IBdata (IBUNAM, 2021) y GBIF (GBIF, 2021). Se enlistan las plantas vasculares con nombres científicos y autores. Se incluyen los nombres de los colectores y números de recolecta para cada ejemplar. Las formas biológicas de las plantas vasculares se indican con $\mathrm{H}=$ hierba; a=arbusto; A=árbol; T=trepadora.

\begin{tabular}{lll}
\hline Taxón & Forma biológica & Colectas \\
\hline
\end{tabular}

\section{PTERIDOFITAS}

Polypodiopsida

Pteridaceae

Cheiloplecton rigidum (Sw.) Fée

\section{ANGIOSPERMAS}

Magnólidas

\section{Annonaceae}

Sapranthus microcarpus (Donn. Sm.) R.E. Fr.

A

a

Piper abalienatum Trel.

\section{Monocotiledóneas}

\section{Asparagaceae}

Agave pablocarrilloi A. Vázquez, Muñiz-Castro \& Padilla-Lepe Bessera elegans Schult. $\mathrm{f}$.

Commelinaceae

Commelina erecta $\mathrm{L}$.

\section{Cyperaceae}

Cyperus esculentus L.

Dioscoreaceae

Dioscorea liebmannii Uline

Dioscorea subtomentosa Miranda

\section{Poaceae}

Cenchrus pilosus Kunth

Panicum trichoides Sw.

Setariopsis auriculata (E. Fourn.) Scribn.

\section{Eudicotiledóneas}

Acanthaceae

Carlowrightia arizonica A. Gray

Justicia caudata A. Gray

\section{Amaranthaceae}

Celosia orcuttii Greenm.

Asteraceae

Lagascea palmeri B.L. Rob.

Montanoa laskowskii McVaugh

Begoniaceae

Begonia monophylla Pourr. ex A. DC.

\section{Bignoniaceae}

J. A. Vázquez G. y J. Padilla L. 9090

R. McVaugh 15552

R. McVaugh 15570

F. García y M. Navarrete 192

W. R. Anderson 12693

O. Téllez V. et al., 10337

M. Navarrete 560

M. Navarrete 553

F.J. Santana M. y N. Cervantes A. 863

R. McVaugh y W. N. Koelz 1590

G. L. Webster y G. J. Breckon 16107

F. J. Santana M. y N. Cervantes A. 873

W. D. Stevens et al., 2558

T. F. Stuessy y M.L. Roberts 3767

R. McVaugh 2557

R. McVaugh y W. N. Koelz 1067 
Apéndice 2. Continuación.

\begin{tabular}{|c|c|c|}
\hline Taxón & Forma biológica & Colectas \\
\hline \multicolumn{3}{|l|}{ Burseraceae } \\
\hline Bursera fagaroides Engl. & A & J. S. Miller y O. Téllez V. 3100 \\
\hline \multicolumn{3}{|l|}{ Capparaceae } \\
\hline Cynophalla verrucosa (Jacq.) J. Presl & A & G. Ibarra 5661 \\
\hline Quadrella indica (L.) H.H. Iltis \& X. Cornejo & A & G. Ibarra et al., 5981 \\
\hline \multicolumn{3}{|l|}{ Celastraceae } \\
\hline Rhacoma sp. & & G. Ibarra 5671 \\
\hline \multicolumn{3}{|l|}{ Convolvulaceae } \\
\hline Ipomoea ternifolia Cav. var. ternifolia & $\mathrm{T}$ & P. Carrillo R. y R. Ramírez D. 3045 \\
\hline Merremia umbellata (L.) Hallier f. & $\mathrm{T}$ & S. D. Koch et al., 89171 \\
\hline \multicolumn{3}{|l|}{ Ehretiaceae } \\
\hline Bourreria superba I.M. Johnst. & A & G. Ibarra et al., 5982 \\
\hline \multicolumn{3}{|l|}{ Erythroxylaceae } \\
\hline Erythroxylum rotundifolium Lunan & A & G. Ibarra 5663 \\
\hline \multicolumn{3}{|l|}{ Euphorbiaceae } \\
\hline Euphorbia cyathophora Murray & $\mathrm{H}$ & G. L. Webster y G. J. Breckon 16118 \\
\hline Euphorbia humayensis Brandegee & $\mathrm{H}$ & G. L. Webster y G. J. Breckon 16114 \\
\hline Euphorbia oaxacana B.L. Rob. \& Greenm. & $\mathrm{T}$ & G. L. Webster y K. I. Miller 16128 \\
\hline Euphorbia schlechtendalii Boiss. var. websteri McVaugh & $\mathrm{T}$ & E. J. Lott y J. A. Solís M. 917 \\
\hline Euphorbia strigosa Hook. \& Arn. & $\mathrm{H}$ & G. L. Webster y G. J. Breckon 16124 \\
\hline Manihot chlorosticta Standl. \& Goldman & $\mathrm{H}$ & R. McVaugh y W. N. Koelz 1553; R. McVaugh 24963 \\
\hline Manihot michaelis McVaugh & A & R. C. Jancey 331, 332, 333 \\
\hline \multicolumn{3}{|l|}{ Fabaceae } \\
\hline Acaciella tequilana (S. Watson) Britton \& Rose & a & F. J. Santana M. y N. Cervantes A. 867 \\
\hline \multirow[t]{2}{*}{ Albizia occidentalis Brandegee } & A & F. J. Santana M. 242 \\
\hline & & J. Maillet 315 \\
\hline Caesalpinia eriostachys Benth. & A & R. McVaugh 22974 \\
\hline Caesalpinia pulcherrima (L.) Sw. & $\mathrm{a}$ & F. J. Santana M. y N. Cervantes A. 501 \\
\hline \multicolumn{3}{|l|}{ Calliandra houstoniana (Mill.) Standl. var. acapulcensis (Britton \& } \\
\hline Rose) Barneby & $\mathrm{a}$ & R. McVaugh 16062 \\
\hline Calliandra tergemina (L.) Benth. & a & J. S. Miller y O. Téllez V. 3098 \\
\hline Cassia hintonii Sandwith & A & R. McVaugh 15576 \\
\hline Coursetia mollis B.L. Rob. \& Greenm. & A & R. McVaugh y W. N. Koelz 1575 \\
\hline Lonchocarpus eriocarinalis Micheli & A & G. Ibarra 5662 \\
\hline Lysiloma microphylla Benth. & A & G. Ibarra et al., 12550 \\
\hline Mimosa caerulea Rose & $\mathrm{H}$ & W. R. Anderson 12695 \\
\hline Mimosa rosei B.L.Rob. & A & O. Téllez V. y A. Novelo 12550 \\
\hline Senna mollissima (Humb. \& Bonpl. ex Willd.) H.S. Irwin \& Barneby & A & J. Maillet JMAI011 \\
\hline Senna villosa (Mill.) H.S. Irwin \& Barneby & a & G. Ibarra et al., 5986 \\
\hline \multicolumn{3}{|l|}{ Lentibulariaceae } \\
\hline Pinguicula colimensis McVaugh \& Mickel & $\mathrm{H}$ & Rzedowski 37805 \\
\hline \multicolumn{3}{|l|}{ Malpighiaceae } \\
\hline Stigmaphyllon sp. & & G. Ibarra 5664 \\
\hline
\end{tabular}


Apéndice 2. Continuación.

\begin{tabular}{|c|c|c|}
\hline Taxón & Forma biológica & Colectas \\
\hline \multicolumn{3}{|l|}{ Malvaceae } \\
\hline Ayenia sp. & & J. Maillet 418 \\
\hline Berrya cubensis (Griseb.) M. Gómez & A & F. J. Santana M. y N. Cervantes A. 240 \\
\hline Gossypium aridum (Rose \& Standl.) Skovst. & A & F. J. Santana M. y N. Cervantes A. 879 \\
\hline Heliocarpus terebinthinaceus (DC.) Hochr. & A & F. J. Santana M. y N. Cervantes A. 869 \\
\hline Waltheria bicolor J.G. Saunders & a & S. D. Koch et al., 89169 \\
\hline \multicolumn{3}{|l|}{ Martyniaceae } \\
\hline Proboscidea fragrans (Lindl.) Decne. & $\mathrm{H}$ & F. J. Santana M. y N. Cervantes A. 502 \\
\hline \multicolumn{3}{|l|}{ Nyctaginaceae } \\
\hline Pisonia aculeata $\mathrm{L}$. & A & M. I. Vergara S. s.n. \\
\hline \multicolumn{3}{|l|}{ Phyllanthaceae } \\
\hline Phyllanthus standleyi McVaugh & $\mathrm{H}$ & G. L. Webster y G. J. Breckon 16129 \\
\hline \multicolumn{3}{|l|}{ Picramniaceae } \\
\hline Alvaradoa amorphoides Liebm. & A & C. D. Johnson 389-73 \\
\hline \multicolumn{3}{|l|}{ Plantaginaceae } \\
\hline Russelia tenuis Lundell & $\mathrm{H}$ & S. D. Koch et al., 89167 \\
\hline \multicolumn{3}{|l|}{ Primulaceae } \\
\hline Bonellia macrocarpa (Cav.) B. Ståhl \& Källersjö & a & G. Ibarra 5667 \\
\hline \multicolumn{3}{|l|}{ Rubiaceae } \\
\hline Chiococca alba (L.) Hitchc. & a & G. Ibarra 5988 \\
\hline Crusea hispida (Mill.) B.L. Rob. var. hispida & $\mathrm{H}$ & W. R. Anderson 12701 \\
\hline Hamelia patens Jacq. & a & G. Ibarra 5657 \\
\hline Randia tetracantha (Cav.) DC. & A & G. Ibarra 5665 \\
\hline \multicolumn{3}{|l|}{ Rutaceae } \\
\hline Zanthoxylum arborescens Rose & A & G. Ibarra 5660 \\
\hline Zanthoxylum melanostictum Schltdl. \& Cham. & A & G. L. Webster y G. J. Breckon 16106 \\
\hline Zanthoxylum mollissimum (Engl.) P. Wilson & A & G. Ibarra 5980 \\
\hline \multicolumn{3}{|l|}{ Urticaceae } \\
\hline Pouzolzia occidentalis (Liebm.) Wedd. & A & G. Ibarra 5658 \\
\hline \multicolumn{3}{|l|}{ Verbenaceae } \\
\hline Citharexylum hexangulare Greenm. & A & G. Ibarra 5984 \\
\hline \multicolumn{3}{|l|}{ Vitaceae } \\
\hline Ampelocissus acapulcensis (Kunth) Planch. & $\mathrm{T}$ & J. S. Miller y O. Téllez V. 3102 \\
\hline Ampelopsis denudata Planch. & $\mathrm{T}$ & J. S. Miller y O. Téllez V. 3099 \\
\hline
\end{tabular}

